\title{
Characteristics and geological significance of tubular carbonate concretions in the Lower Shaximiao Formation of Yunyang, Chongqing, China
}

\author{
${ }^{1}$ Institute of Sedimentary Geology, Chengdu University of Technology, Chengdu, 610059, China; *Corresponding author, E-mail: yangwg1018@gmail.com \\ ${ }^{2}$ College of Earth Science, Chengdu University of Technology, Chengdu, 610059, China
}

(Received: January 15, 2021; Revised accepted: April 29, 2021)

https://doi.org/10.18814/epiiugs/2021/021005

Methane-derived carbonates provide strong evidence supporting the emission of unstable gas-hydrates from the seafloor. However, existing studies have shown that methane-derived carbonates are mainly formed in underwater marine environments, and there are few reports regarding their formation in terrestrial basins. A large number of nodular and tubular concretions were developed in the Middle Jurassic Shaximiao Formation in Yunyang, Chongqing. This paper focuses on the two types of calcareous concretions, and their detailed macroscopic, microscopic and geochemical characteristics are described. Our results indicate that the observed nodular concretions were the product of continuous evaporation under arid-semiarid conditions. The morphologies, microscopic features and carbon and oxygen isotope compositions of the tubular concretions are similar to those of tubular methane-derived carbonates. Therefore, we believe that the tubular concretions in the study area are related to methane emissions and that they acted as channels for the migration of methane-rich fluids and muddy sediments. Based on the background of sediment liquefaction in the study area, we suggest that these tubular concretions might be the most direct geological records of mud volcanic activity.

\section{Introduction}

As a common sedimentary structure in sedimentary rocks, different types of calcareous concretions record the geological information of different formation environments (Pang et al., 2017). Concretions can be divided into syngenetic, diagenetic, and epigenetic concretions (Pantin, 1958) according to the different formation stages, among which research data on concretions formed diagenetically are the most abundant due to their wide distribution. Methane leakage is considered one of the main formation mechanisms of early diagenetic calcareous concretions (Clari et al., 2004; Tong and Chen, 2012; Ou et al., 2013; Liang et al., 2016). Tubular concretions are the most common prod- uct of seafloor methane leakage and are often used as migration channels for underground fluids and liquefied sediments (Nyman et al., 2010). These channels the main evidence to supporting the identification of ancient seafloor methane leakage and even mud volcano activities (Aiello et al., 2001; Campbell, 2002; Schwartz et al., 2003; Clari et al., 2004; Liang et al., 2016). Methane leakage can also form concretions in the shape of mounds, nodules, clintheriforms, chimneys and many other features (Peckmann et al., 2001; Conti and Fontana, 2005; Pierre et al., 2010).

Due to the unique carbon source of calcareous concretions resulting from methane leakage and the fact that the carbon isotopes of carbonate minerals are less affected by diagenesis, this type of carbonate has an extremely low $\delta^{13} \mathrm{C}$ composition (Whiticar et al., 1986). However, because of the complexity of geologic processes, the formation of authigenic carbonates may be the result of the mixing of multiple carbon sources. The degree of mixing of various carbon sources is not clear, although the carbon sources can still be qualitatively evaluated according to the $\delta^{13} \mathrm{C}$ contents of samples (Peckmann and Thiel, 2004).

The reported hydrocarbon leakage sources and hydrothermal vents were believed to be mainly distributed along plate boundaries (midocean ridges and subduction zones), back-arc basins, and areas with a large number of fractures and diapirs worldwide until now and are mostly located in marine underwater environments (Fig. 1a) (Campbell, 2006). These calcareous concretions formed by methane leakage have attracted increasing attention from scholars worldwide due to the close relationship between methane leakage and undersea natural gas hydrates. It is not difficult to summarize previous studies and determine that there are few reports of methane-derived authigenic carbonates in terrestrial basins.

In this paper, we report tubular concretions distributed in the sediments of the Lower Member of the Shaximiao Formation in the northeastern part of the Sichuan Basin. These tubular concretions have a typical channel structure inside, which may be characteristic of concretions derived from methane leakage. Here, the morphology, petrography, elemental composition, and isotopic geochemistry of samples were analyzed to explore the genesis and geological significance of tubular nodules in the study area. 


\section{Geological Setting}

Located west of the South China plate, the Sichuan Basin is surrounded by fold-and-thrust belts (Guo et al., 1996; Meng et al., 2005; Li et al., 2018a). The Sichuan Basin has been in a stage of terrestrial basin evolution since the end of the Triassic, and strata filling mainly occurred during the Jurassic with fluvial and lacustrine deposits (Fig. 1b). The study area of this study is located in the northeastern part of the Sichuan Basin, belonging to Pu'an Township, Yunyang, Chongqing. The Jurassic strata exposed in this area include the Lower Jurassic Ziliujing Formation $\left(\mathrm{J}_{1} z\right)$, the Middle Jurassic Xintiangou Formation $\left(\mathrm{J}_{2} x t\right)$, the Middle Jurassic Shaximiao Formation $\left(\mathrm{J}_{2} \mathrm{~s}\right)$, the Upper
Jurassic Suining Formation $\left(\mathrm{J}_{3} s n\right)$, the Upper Jurassic Penglaizhen Formation $\left(\mathrm{J}_{3} p\right)$ and a small amount of Triassic strata (Fig. 1c).

In the Early Jurassic, the Sichuan Basin was dominated by stable lacustrine deposits (Wang and $\mathrm{Xu}, 2001$ ), and the dark argillaceous shales of the Da'anzhai Member of the Lower Jurassic Ziliujing Formation are considered important oil-producing beds in basin, which contains rich oil and gas resources and has great potential for hydrocarbon generation (Chen et al., 2020; Zhang et al., 2020). As the main Middle Jurassic sediment sources in the area, the Xintiangou Formation and Shaximiao Formation represent typical lake delta and river delta deposits, respectively (Chen et al., 2019).

The Shaximiao Formation in this area can be divided into Upper and Lower Members by gray-black shale with conchostracan fauna.
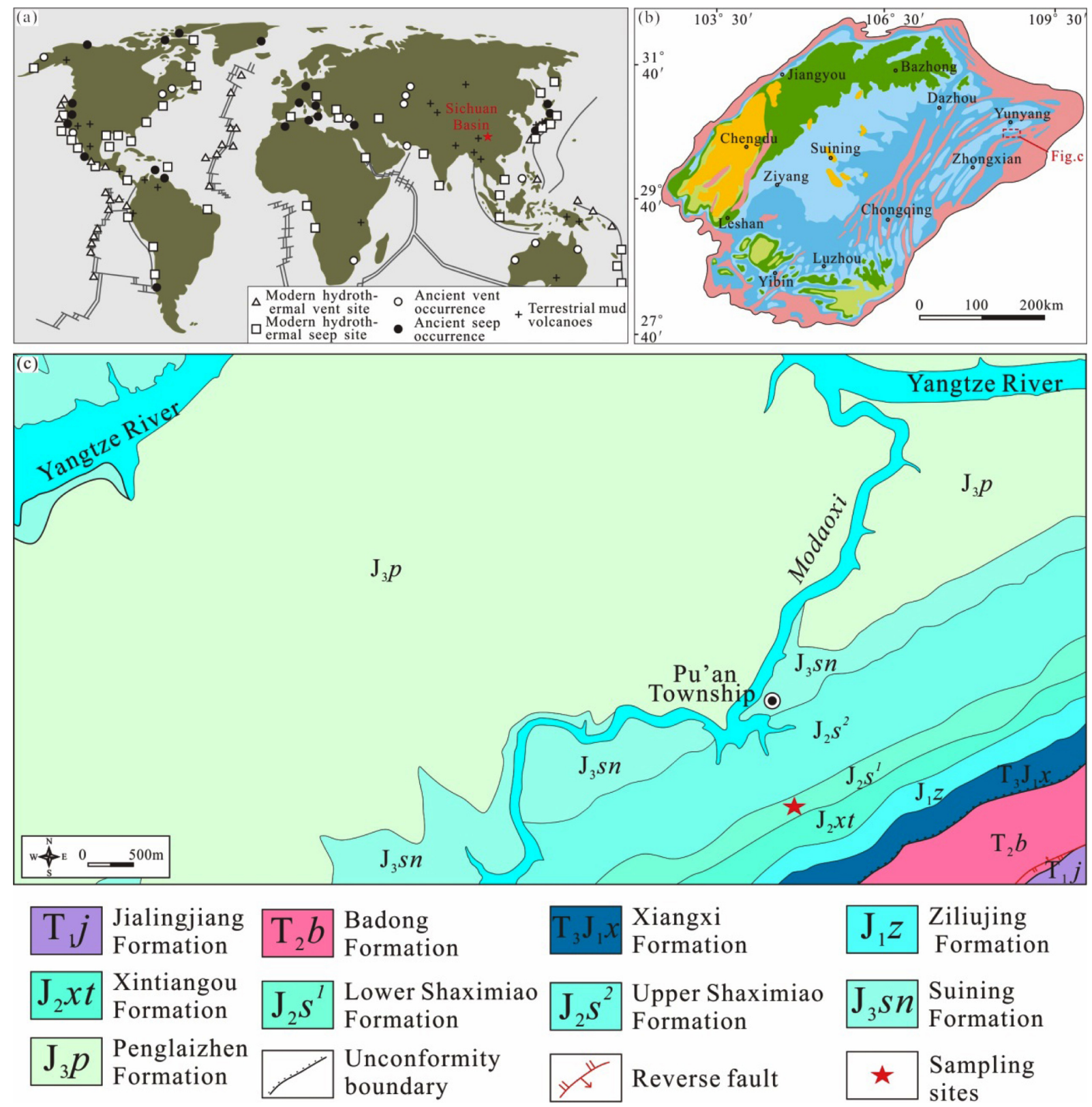

Figure 1. Geographical location map and simplified geological maps. (a) Global distribution of reported modern and ancient cold methane seeps and hydrothermal vent sites (modified from Mikov, 2005 and Campbell, 2006), (b) Simplified geological map of the Sichuan Basin (modified from Li et al., 2018a), and (c) Geological map of the study area (modified from 1:50,000 regional geological survey reports of Yunyang County, Chongqing Municipality, China). 


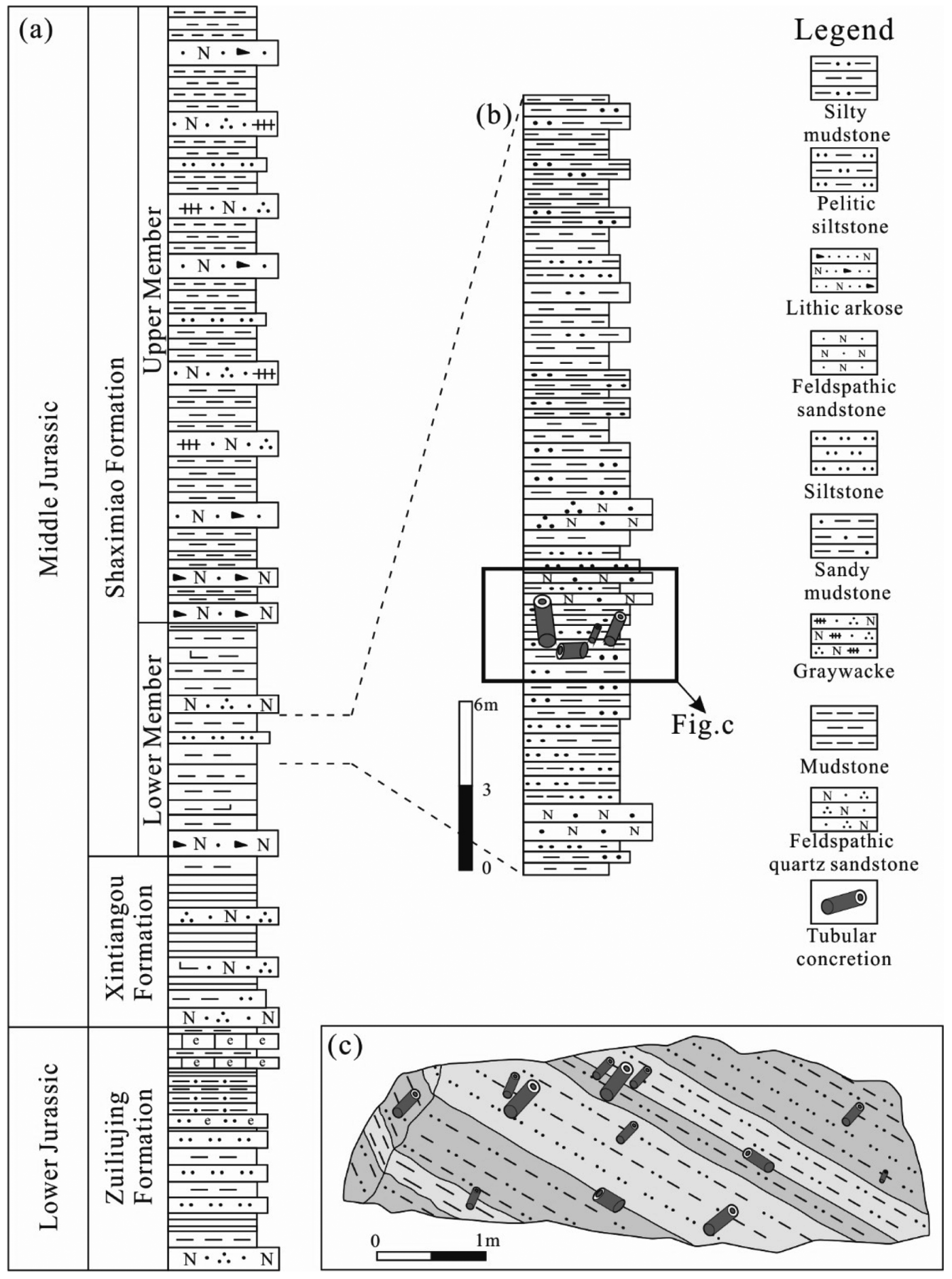

Figure 2. Stratigraphic section of the sedimentary sequence of the study area. (a) The Jurassic strata in the study area, (b) Subtle section of Lower Shaximiao Formation, and (c) Sketch map of the tubular concretions and surrounding rocks (modified from Clari et al., 2004).

The Lower Shaximiao Formation is dominated by purple-red and gray thin mudstone and argillaceous siltstone and occasionally contains several sets of thick feldspar quartz sandstone (Fig. 2a). Tubular concretions occur in the middle part of the Lower Shaximiao Formation, and the surrounding rocks are mainly gray and purplish-red siltstones (Figs. 2b and 2c). These concretions are tubular and columnar in shape and oblique (nearly vertical) to the surrounding rock strata bedding at a high angle, and a few are flat and extend along the rock strata (Figs. 2c and 3a). Generally, tubular concretions feature a pipeline structure, and the conduits are fully filled with brown fine sediments and calcites.

\section{Samples and Methods}

According to field observations, concretions in the Shaximiao For- 
mation in the study area mainly include two types: nodular and tubular. This study mainly focuses on those tubular concretions. Therefore, 15 tubular concretions were collected. At the same time, to explore their genesis, relevant experiments were carried out for nodular concretions (3 samples) in the Upper Shaximiao Formation and limestone (3 samples) from the top of the Lower Jurassic Ziliujing Formation. These samples were cut transversally for thin section preparation and observation under a microscope. Additionally, some of the samples were polished for internal structure observation. Meanwhile, the analysis of major and trace elements and stable carbon and oxygen isotopes was carried out.

The major, trace, and rare earth elements of samples were determined in Guangzhou Aoshi Mineral Laboratory. The major elements were tested by X-ray fluorescence spectrometry (ME-XRF26D), and the rare earth elements were tested by plasma mass spectrometry
(ME-MS81). Integrated mass spectrometry analysis was carried out for trace element identification (ME-MS61). Geochemical data processing and graphing were completed in GeoPlot and other software.

Stable carbon and oxygen isotope analyses were finished in the laboratory of Beijing Createch Testing Co., Ltd. The equipment used was the 253 Plus and Gas Bench of Seymour Fisher. The temperature of the chromatographic column (Poraplot Q, $25 \mathrm{~m} \times 0.32 \mathrm{~mm}$ ) was $70^{\circ} \mathrm{C}$. Approximately $100 \mathrm{~g}$ of the carbonate samples were weighed (200 mesh) and added into a $12 \mathrm{~mL}$ reaction bottle. High purity helium gas was used for emptying, and then 5 drops of $100 \%$ anhydrous phosphoric acid were added and placed in a $72{ }^{\circ} \mathrm{C}$ heating dish for reaction and balance completion. After the sample reacted with phosphoric acid and reached equilibrium, $\mathrm{CO}_{2}$ gas was separated from other impurities and then determined by gas stable isotope mass spectrometry. The analytical precision is higher than $0.1 \%$.
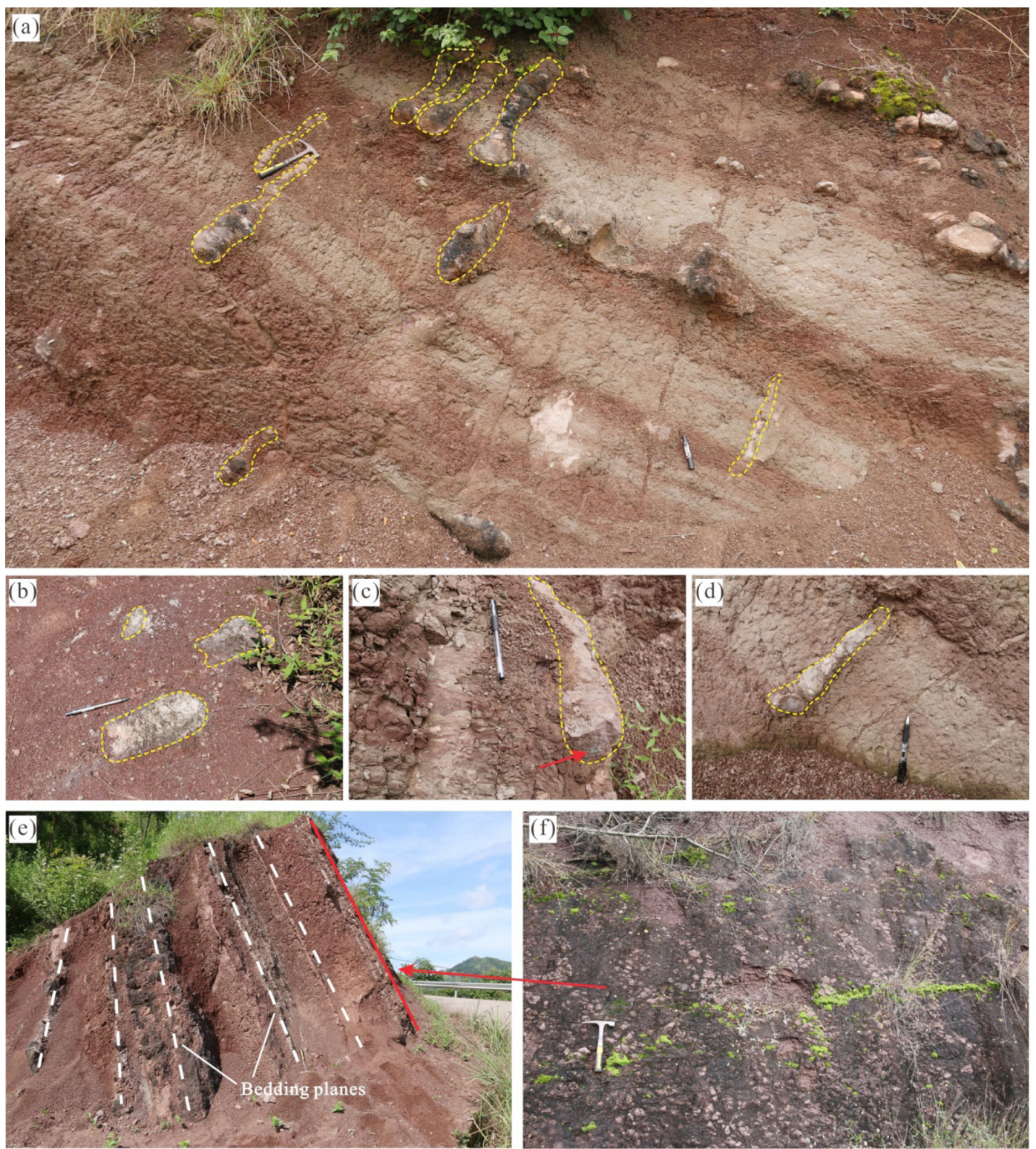

Figure 3. Field characteristics of calcareous concretions. (a) Tubular concretions, (b) Weathered tubular concretions, (c) Tubular concretions were filled with sediments internally (red arrow), (d) Tubular concretions were perpendicular to the rock bedding, (e) The nodular concretions were parallel to the stratigraphic bedding, and (f) A local enlargement of nodular concretions. 


\section{Results}

\section{Morphology and Petrography}

The tubular concretions are light purple to red in color, and the shapes are mostly cylindrical or beaded (Fig. 3). Some are enlarged and narrowed obviously, forming a sac with a thick bottom and narrow top (Fig. 3a). Different concretions vary in size, shorter samples may reach approximately $20 \mathrm{~cm}$ in length, while the longer samples can reach $1-2 \mathrm{~m}$, and the diameters are approximately $3-25 \mathrm{~cm}$. The tubular calcareous nodules obliquely intersect with the rock bed at a high angle (nearly vertical) and may connect to the horizontal sandy beds or be distributed independently. Some of those tubular concretions exhibit uniform lithology, compactness, and development without inner texture. However, most have bluish-gray calcareous cemented sand within the inner cores, appearing in the shape of conduits. Meanwhile, a small number of flat ellipsoid-shaped and tubular concretions developed with each other. Their diameters range from centimeters to tens of centimeters. Nodular concretions are widely distributed in the study area and are mainly developed in the Upper Shaximiao Formation. Those layered nodular concretions (the white dotted line in Fig. $3 \mathrm{e}$ ) are parallel to the stratification planes, which is different from the characteristics of the tubular concretions. Macroscopically, these concretions are nodular in shape, and a large amount of nodules are gathered together and cover the surface of the rock like a carpet (Fig. 3f).

Macroscopically, calcite veins are distributed in a fissured shape inside the nodular concretions, and those veins are irregular and mostly dendritic with poor extensibility. The veins converge and become thinner from the center to the edge (Fig. 4a). Different from nodular concretions, tubular concretions show obvious pipe-like structures inside (Figs. 4b-f), and the cross-sections of those conduits usually exist in a layered form of nearly concentric circles with diameters of several millimeters to several centimeters. The conduits are mainly filled with different materials, calcites, and argillaceous sediments and detrital matrix (red arrows). Calcite filling fissures may appear inside the tubular concretions (green arrows in Fig. 4d), which are distributed in a nearly radial pattern. The longitudinal sections show the inner texture inside the tubular concretions (Figs. $4 \mathrm{e}$ and $4 \mathrm{f}$ ), and the fillings are white calcites and dark muddy sediments. Fig. $4 \mathrm{f}$ shows the role of
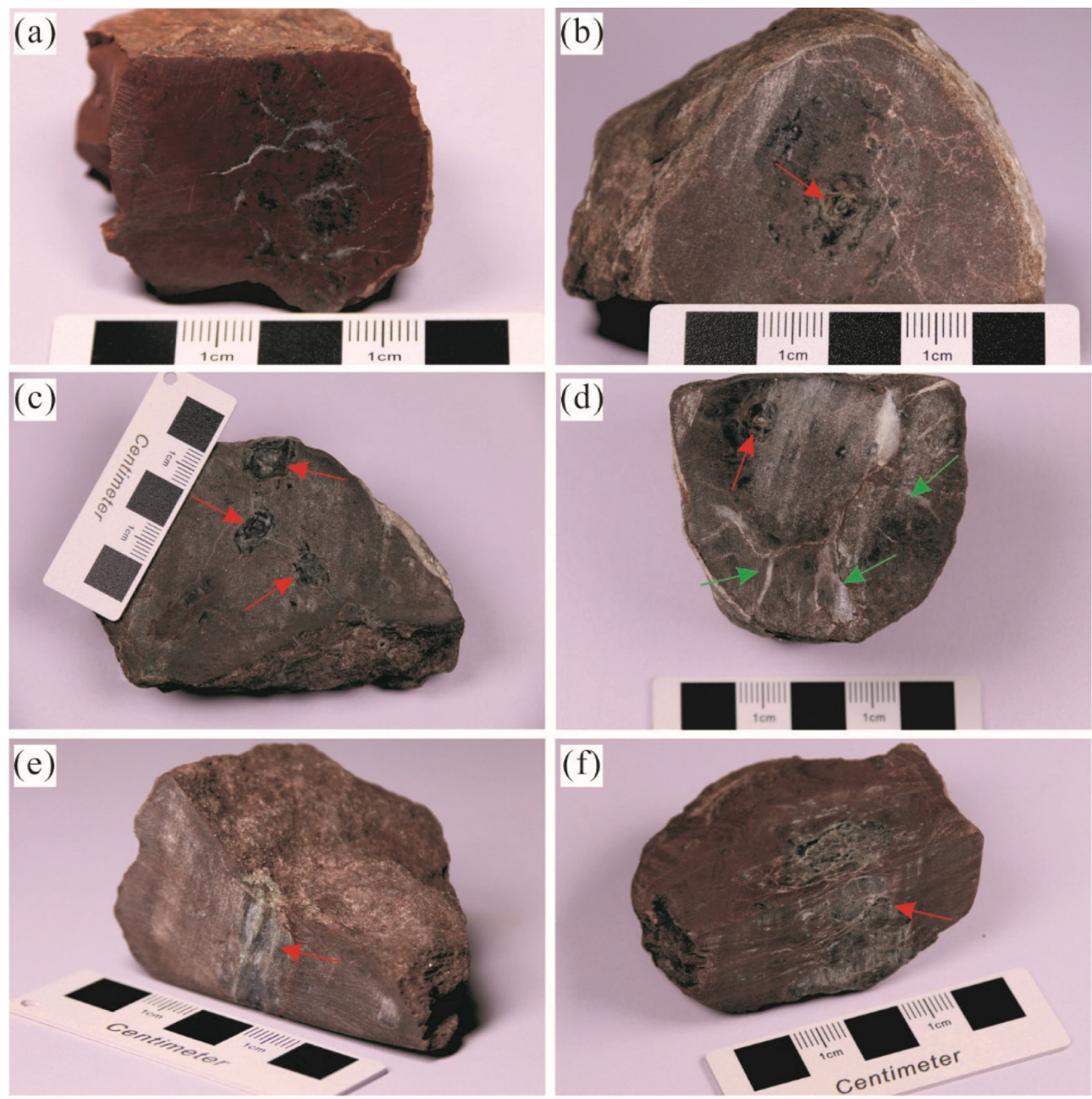

Figure 4. Macroscopic photos of calcareous concretions. (a) Nodular concretion filled with calcite veins internally, (b, c) Circle-layered core of a tubular concretion (red arrows), (d) Circle-layered core of tubular concretions (red arrow) and radial fissures. These fissures were filled with calcites (green arrows), (e) Vertical section of a tubular concretion, and (f) Tubular nodules act as transport channels for argillaceous materials, and the weak traction deformation of the "surrounding rock". 


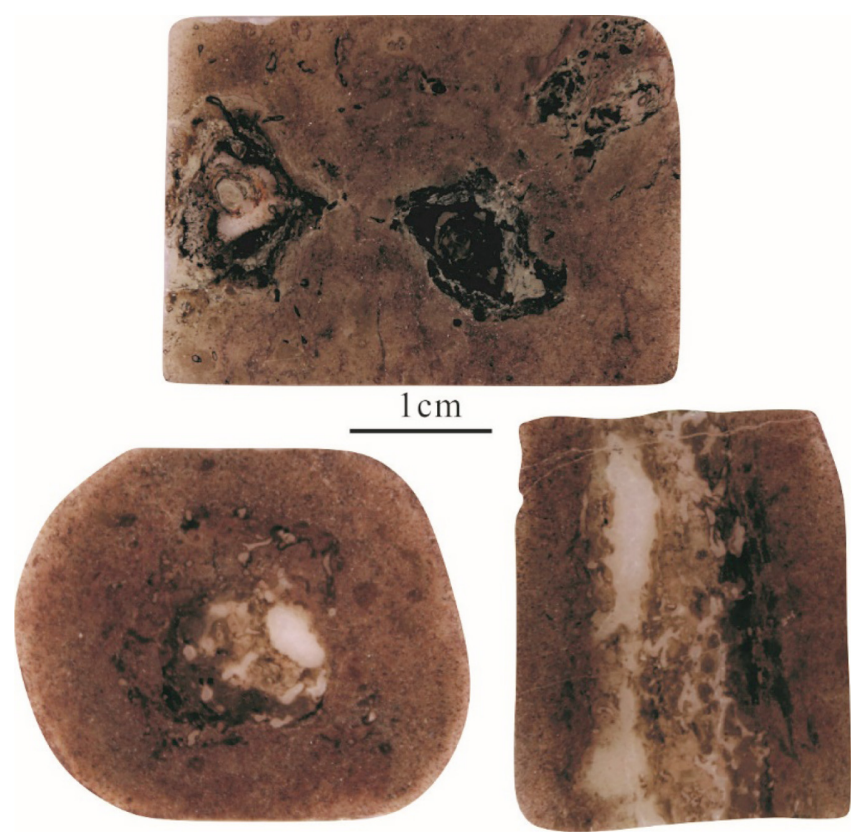

Figure 5. Two transverse slabs and one axial polished slab of calcareous concretions.

the pipeline as a channel for the upwelling of sandy muddy sediments. The later filling sediments migrated in the conduit formed earlier, causing the pipe wall to deform weakly. In enlarged polished slabs tubular concretions, the axial portion is filled with sediment, mainly dark gray cemented mud and light-colored crystallographic calcites (Fig. 5).

Viewing the features under a microscope reveals that the tubular concretions are mainly filled with calcites and sandy argillaceous debris.
The ring-like texture is highlighted by different materials. The internal fillings mainly include anhedral sparry calcites (AC) and micrite (M), and they are surrounded by a cemented sandy clastic matrix (m) (Fig. 6a). An enlarged view shows that the inner core of the pipeline is mainly composed of radial fibrous calcites (FC) and fine granular calcites (GC) (Fig. 6b). From the anhedral sparry calcites (AC) shown in Fig. 6c, in combination with the characteristics shown in Figs. 6d and $6 \mathrm{e}$, we conclude that these anhedral sparry calcites (AC) were formed much later than the lithification of those concretions, they formed during the late diagenetic stage and later than the other material in the conduit. In some fields of vision, the fillings are argillaceous sediments, and the argillaceous materials also possess cyclic annular shapes. A small amount of well-crystallized calcite can be seen in the right center of the conduit (Fig. 6d). Some fillings within the conduit are mainly composed of cemented sandy debris of surrounding rocks (Fig. 6e). Additionally, a large number of (elliptical) round calcite filling textures can be seen under the microscope (Fig. 6f), and the calcites inside are well-crystallized. Fibrous calcites (FC) (red arrow) and anhedral calcites (AC) are placed in order from the edge to the center, and the outer borders of the texture are surrounded by a dark brown ring (green arrow). In general, the internal channel tube of the tubular concretions is nearly circular, and the channel tube is filled with sediments of different compositions and structures, showing a clear layered structure.

\section{Geochemistry}

The results of whole-rock geochemistry show that the elements of the nodular and tubular concretions and limestone samples from the top of the Ziliujing Formation have no noteworthy differences among
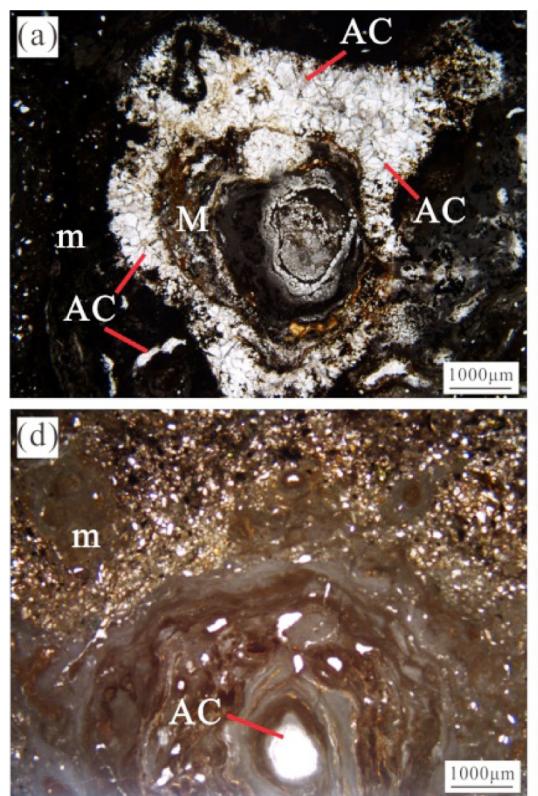
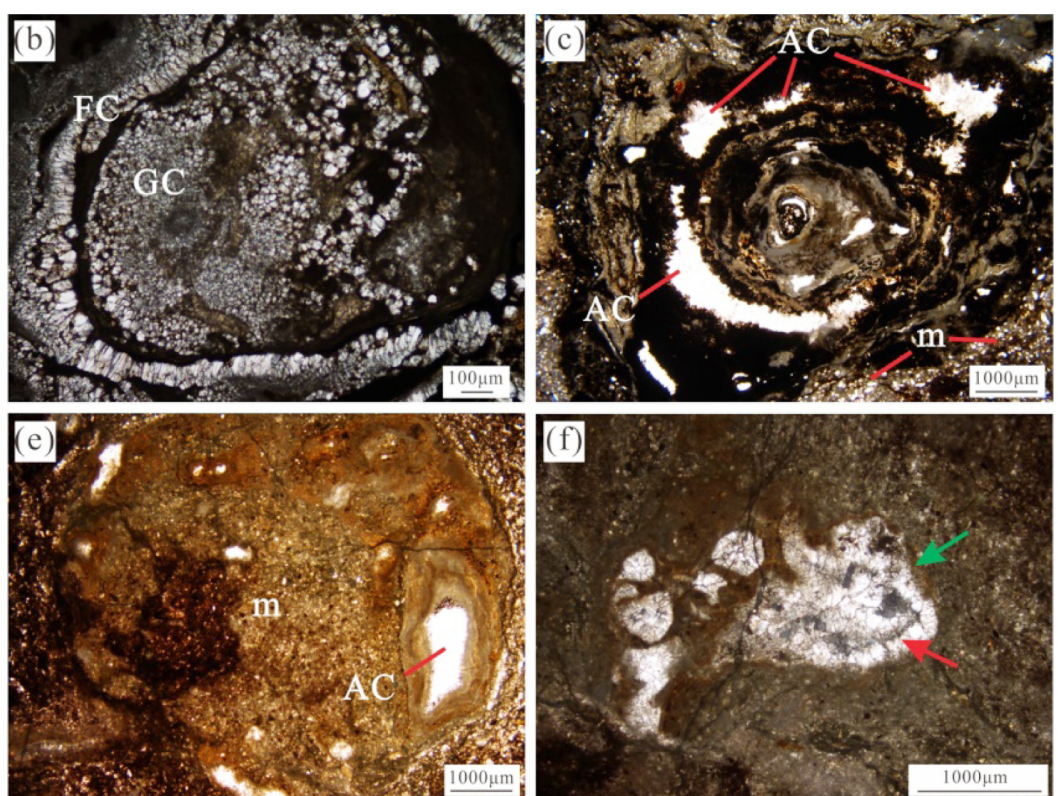

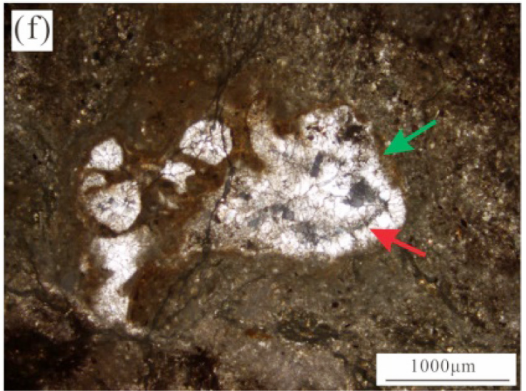

Figure 6. Microscopic characteristics of tubular calcareous concretions. (a) Pipe structure consisting of anhedral calcites (AC), micrite (M) and matrix (m), (b) Local enlarged view of image a, showing fibrous calcites (FC) and granulous calcites (GC), (c) Pipe structure consisting of anhedral calcites $(A C)$ and pelytes, (d) Argillaceous fillings and anhedral calcites $(A C)$ in the center, (e) The conduits were filled with matrix (m), and $(f)$ View of spheres showing the dark rim (green arrow) and the sparry internal fabric; note that the inner core was filled with fibrous calcites (FC) (red arrow) and anhedral calcites (AC) from the edge to center. AC-Anhedral Calcites, FC-Fibrous Calcites, GCGranulous Calcites, M-Micrite, m-matrix. 
Table 1. Major (\%) element analyzing results of samples

\begin{tabular}{|c|c|c|c|c|c|c|c|c|c|c|c|c|}
\hline Sample No. & Types & $\mathrm{SiO}_{2}$ & $\mathrm{TiO}_{2}$ & $\mathrm{Al}_{2} \mathrm{O}_{3}$ & $\mathrm{Fe}_{2} \mathrm{O}_{3} \mathrm{~T}$ & $\mathrm{MnO}$ & $\mathrm{MgO}$ & $\mathrm{CaO}$ & $\mathrm{Na}_{2} \mathrm{O}$ & $\overline{\mathrm{K}} \mathrm{K}_{2} \mathrm{O}$ & $\mathrm{P}_{2} \mathrm{O}_{5}$ & LOI \\
\hline Y016-DH1 & Nodular concretion & 29.42 & 0.38 & 7.96 & 3.22 & 0.36 & 1.67 & 28.9 & 0.74 & 1.77 & 0.12 & 24.95 \\
\hline Y039-DH1 & Nodular concretion & 22.35 & 0.29 & 5.9 & 2.44 & 0.59 & 1.21 & 35.3 & 0.67 & 1.12 & 0.1 & 29.13 \\
\hline Y049-DH1 & Nodular concretion & 19.6 & 0.23 & 6.35 & 2.23 & 0.5 & 1.09 & 36.8 & 0.28 & 1.42 & 0.29 & 30.62 \\
\hline Mean & & 23.79 & 0.30 & 6.74 & 2.63 & 0.48 & 1.32 & 33.67 & 0.56 & 1.44 & 0.17 & 28.23 \\
\hline Y103-DH3 & Limestone & 1.24 & 0.01 & 0.35 & 0.25 & 0.01 & 0.1 & 54.3 & 0.03 & 0.07 & 0.11 & 42.81 \\
\hline Y103-DH4 & Limestone & 5.27 & 0.05 & 2.03 & 0.65 & 0.01 & 0.14 & 50.7 & 0.03 & 0.33 & 0.03 & 39.94 \\
\hline Mean & & 3.26 & 0.03 & 1.19 & 0.45 & 0.01 & 0.12 & 52.50 & 0.03 & 0.20 & 0.07 & 41.38 \\
\hline Y002-DH1 & Tubular concretion & 47.84 & 0.49 & 11.36 & 4.14 & 0.41 & 1.67 & 16 & 1.88 & 1.53 & 0.12 & 14.85 \\
\hline Y002-DH2 & Tubular concretion & 41.61 & 0.46 & 10.14 & 3.54 & 0.48 & 1.49 & 20.7 & 1.57 & 1.44 & 0.14 & 18.32 \\
\hline Y002-DH3 & Tubular concretion & 33.63 & 0.36 & 8.04 & 2.55 & 0.54 & 1.28 & 27 & 1.28 & 1.22 & 0.22 & 23.01 \\
\hline Y002-DH4 & Tubular concretion & 34.33 & 0.36 & 8.22 & 2.55 & 0.46 & 1.45 & 26.5 & 1.31 & 1.23 & 0.16 & 22.65 \\
\hline Y002-DH5 & Tubular concretion & 41.09 & 0.42 & 9.68 & 3.35 & 0.62 & 1.47 & 21.3 & 1.6 & 1.29 & 0.13 & 18.75 \\
\hline Y002-DH6 & Tubular concretion & 37.36 & 0.46 & 9.84 & 3.19 & 0.54 & 1.53 & 23.3 & 1.31 & 1.5 & 0.54 & 20.18 \\
\hline Y002-DH7 & Tubular concretion & 34.79 & 0.36 & 8.32 & 2.59 & 0.51 & 1.41 & 26.3 & 1.3 & 1.21 & 0.14 & 22.46 \\
\hline Y002-DH8 & Tubular concretion & 40.02 & 0.46 & 9.71 & 3.05 & 0.47 & 1.4 & 22.3 & 1.36 & 1.53 & 0.22 & 19.48 \\
\hline Y002-DH9 & Tubular concretion & 34.84 & 0.42 & 8.94 & 2.87 & 0.48 & 1.3 & 25.9 & 1.28 & 1.39 & 0.19 & 22.19 \\
\hline Y002-DH10 & Tubular concretion & 37.9 & 0.44 & 9.63 & 3.19 & 0.56 & 1.46 & 23.2 & 1.32 & 1.44 & 0.18 & 20.42 \\
\hline Y002-DH11 & Tubular concretion & 38.24 & 0.45 & 9.43 & 3.04 & 0.63 & 1.42 & 23.1 & 1.25 & 1.47 & 0.16 & 20.55 \\
\hline Y002-DH12 & Tubular concretion & 37.47 & 0.42 & 9.15 & 3.25 & 0.57 & 1.49 & 23.9 & 1.31 & 1.38 & 0.15 & 20.76 \\
\hline Y002-DH13 & Tubular concretion & 37.22 & 0.45 & 9.53 & 3.25 & 0.59 & 1.38 & 23.8 & 1.3 & 1.44 & 0.2 & 20.61 \\
\hline Y002-DH14 & Tubular concretion & 42.7 & 0.52 & 11.24 & 4.23 & 0.58 & 1.73 & 18.45 & 1.58 & 1.63 & 0.19 & 16.81 \\
\hline Y002-DH15 & Tubular concretion & 38.36 & 0.49 & 10.2 & 3.71 & 0.67 & 1.58 & 22 & 1.33 & 1.54 & 0.2 & 19.41 \\
\hline Mean & & 38.49 & 0.44 & 9.56 & 3.23 & 0.54 & 1.47 & 22.92 & 1.40 & 1.42 & 0.20 & 20.03 \\
\hline
\end{tabular}

the same category of samples, but the three are quite different from each other (Table 1). The contents of $\mathrm{TiO}_{2}, \mathrm{MnO}, \mathrm{MgO}, \mathrm{Na}_{2} \mathrm{O}, \mathrm{K}_{2} \mathrm{O}$, and $\mathrm{P}_{2} \mathrm{O}_{5}$ are extremely low in all samples. High $\mathrm{SiO}_{2}$ and $\mathrm{CaO}$ contents and a high loss on ignition (LOI) account for a large proportion of the samples, and there are obvious differences between different sample types (Fig. 7). Limestone contains a small amount of $\mathrm{SiO}_{2}$, and the average value of the two samples is approximately $3.27 \%$, while $\mathrm{SiO}_{2}$ accounts for 23.79 and $38.49 \%$ (on average) of nodular and tubular concretions, respectively. The average $\mathrm{CaO}$ concentration of the limestone from the Ziliujing Formation is approximately $52.5 \%$. Approximately $33.67 \%$ of the samples exhibited nodular concretion, and $22.92 \%$ exhibited tubular concretion. Among the three types of samples, their

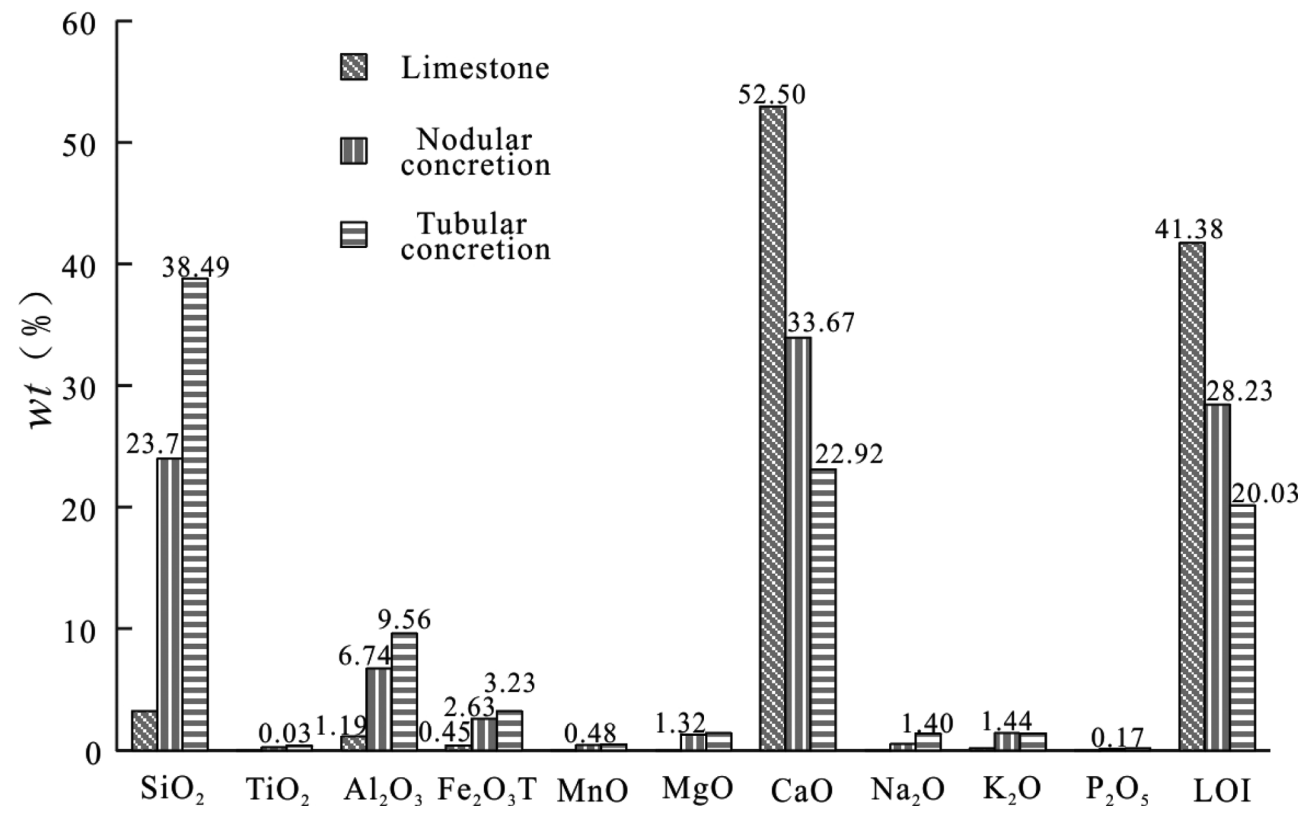

Figure 7. Column diagrams of major elements for concretions and limestones (average values). 

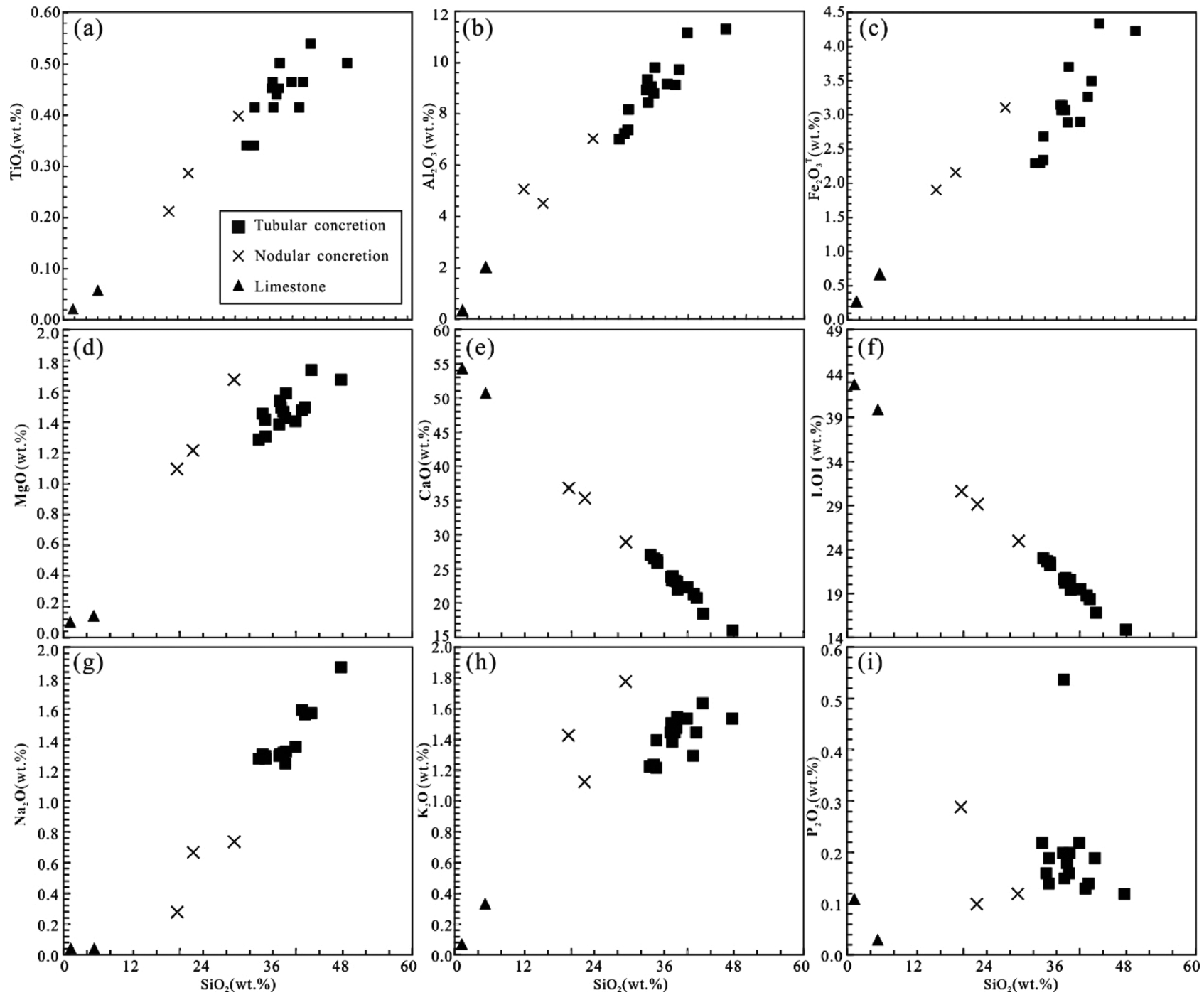

Figure 8. Binary diagrams of the correlations of major elements.

losses on ignition (LOI) account for $41.38,28.23$, and $20.03 \%$, respectively (Fig. 7). Specifically, excluding some oxides $\left(\mathrm{K}_{2} \mathrm{O}\right.$ and $\left.\mathrm{P}_{2} \mathrm{O}_{5}\right)$, most of the oxides in the samples have an obvious linear relationship with the $\mathrm{SiO}_{2}$ contents of the rocks (Fig. 8). Among them, $\mathrm{TiO}_{2}, \mathrm{Al}_{2} \mathrm{O}_{3}$,

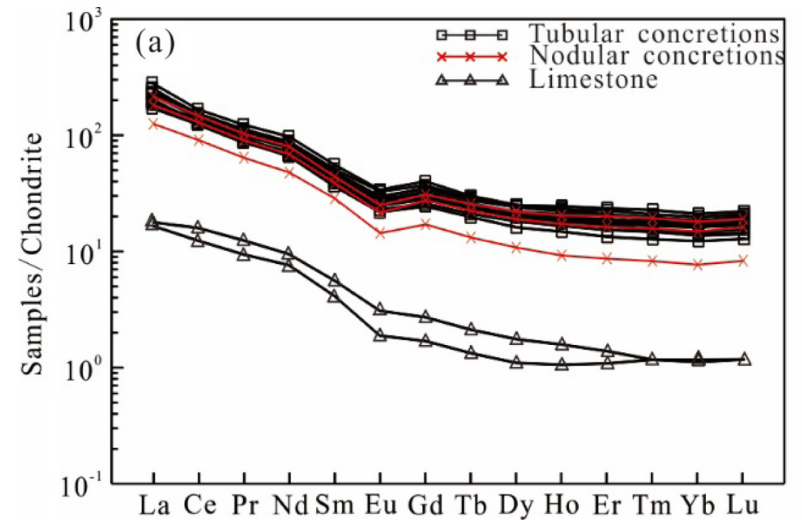

$\mathrm{Fe}_{2} \mathrm{O}_{3}{ }^{\mathrm{T}}, \mathrm{MgO}, \mathrm{Na}_{2} \mathrm{O}$, etc. are positively correlated with the $\mathrm{SiO}_{2}$ content. However, $\mathrm{CaO}$ and loss on ignition (LOI) have a consistent negative correlation with $\mathrm{SiO}_{2}$, and $\mathrm{K}_{2} \mathrm{O}$ and $\mathrm{P}_{2} \mathrm{O}_{5}$ are not significantly correlated.

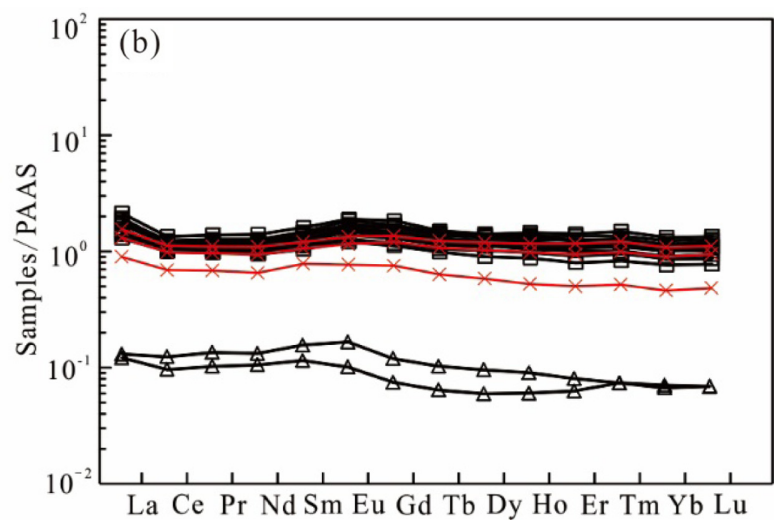

Figure 9. Partitioning patterns of rare-earth elements (REEs) for samples. (a) Normalized to chondrites, and (b) Normalized to Australian Post-Archean Shales (PAAS). 
Table 2. Trace(ppm) element analyzing results of samples

\begin{tabular}{|c|c|c|c|c|c|c|c|c|c|c|c|}
\hline \multicolumn{2}{|c|}{ Sample No. } & \multirow{2}{*}{$\begin{array}{c}\text { Y016-DH1 } \\
0.08\end{array}$} & \multirow{2}{*}{$\begin{array}{c}\text { Y039-DH1 } \\
0.03\end{array}$} & \multirow{2}{*}{$\begin{array}{c}\text { Y049-DH1 } \\
0.05\end{array}$} & \multirow{2}{*}{ 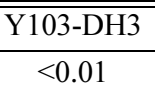 } & \multirow{2}{*}{$\begin{array}{c}\text { Y103-DH4 } \\
0.01\end{array}$} & \multirow{2}{*}{$\begin{array}{c}\text { Y002-DH1 } \\
0.06\end{array}$} & \multirow{2}{*}{$\begin{array}{c}\text { Y002-DH2 } \\
0.06\end{array}$} & \multirow{2}{*}{$\begin{array}{c}\text { Y002-DH3 } \\
0.09\end{array}$} & \multirow{2}{*}{$\begin{array}{c}\text { Y002-DH4 } \\
0.06\end{array}$} & \multirow{2}{*}{$\begin{array}{c}\text { Y002-DH5 } \\
0.07\end{array}$} \\
\hline $\mathrm{Ag}$ & $\mu \mathrm{g} / \mathrm{g}$ & & & & & & & & & & \\
\hline $\mathrm{Al}$ & $\%$ & 4.36 & 3.24 & 3.47 & 0.22 & 1.11 & 5.91 & 5.28 & 4.31 & 4.27 & 5.17 \\
\hline As & $\mu \mathrm{g} / \mathrm{g}$ & 11.3 & 6.6 & 17.2 & 9.8 & 5.7 & 6.4 & 7.6 & 5.9 & 5.7 & 6.9 \\
\hline $\mathrm{Ba}$ & $\mu \mathrm{g} / \mathrm{g}$ & 356 & 756 & 270 & 161.5 & 165.0 & 430 & 378 & 376 & 474 & 651 \\
\hline $\mathrm{Be}$ & $\mu \mathrm{g} / \mathrm{g}$ & 1.69 & 1.19 & 1.77 & 0.14 & 0.33 & 1.62 & 1.51 & 1.37 & 1.38 & 1.42 \\
\hline $\mathrm{Bi}$ & $\mu \mathrm{g} / \mathrm{g}$ & 0.22 & 0.11 & 0.18 & 0.02 & 0.03 & 0.11 & 0.10 & 0.09 & 0.09 & 0.09 \\
\hline $\mathrm{Ca}$ & $\%$ & 21.1 & 25.5 & 26.4 & 38.1 & 35.0 & 11.00 & 14.30 & 19.35 & 18.30 & 15.25 \\
\hline $\mathrm{Cd}$ & $\mu \mathrm{g} / \mathrm{g}$ & 1.94 & 1.70 & 1.18 & 0.02 & 0.04 & 1.27 & 1.23 & 2.23 & 2.31 & 1.98 \\
\hline $\mathrm{Ce}$ & $\mu \mathrm{g} / \mathrm{g}$ & 77.9 & 88.7 & 54.9 & 9.9 & 7.7 & 81.7 & 84.8 & 82.9 & 80.4 & 83.4 \\
\hline $\mathrm{Co}$ & $\mu \mathrm{g} / \mathrm{g}$ & 9.7 & 7.5 & 7.4 & 2.8 & 3.7 & 11.0 & 10.5 & 8.3 & 9.1 & 10.0 \\
\hline $\mathrm{Cr}$ & $\mu \mathrm{g} / \mathrm{g}$ & 34 & 24 & 27 & 6 & 9 & 39 & 40 & 36 & 43 & 41 \\
\hline Cs & $\mu \mathrm{g} / \mathrm{g}$ & 4.59 & 3.01 & 4.50 & 0.25 & 1.10 & 3.78 & 3.67 & 3.41 & 3.60 & 3.42 \\
\hline $\mathrm{Cu}$ & $\mu \mathrm{g} / \mathrm{g}$ & 19.1 & 11.6 & 12.8 & 5.6 & 7.2 & 15.0 & 14.7 & 13.9 & 13.3 & 13.7 \\
\hline Dy & $\mu \mathrm{g} / \mathrm{g}$ & 4.78 & 5.57 & 2.73 & 0.45 & 0.28 & 5.24 & 5.28 & 5.53 & 5.74 & 5.24 \\
\hline $\mathrm{Er}$ & $\mu \mathrm{g} / \mathrm{g}$ & 2.67 & 3.31 & 1.43 & 0.23 & 0.18 & 2.86 & 2.82 & 3.26 & 3.56 & 2.99 \\
\hline $\mathrm{Eu}$ & $\mu \mathrm{g} / \mathrm{g}$ & 1.24 & 1.44 & 0.83 & 0.18 & 0.11 & 1.60 & 1.68 & 1.71 & 1.64 & 1.60 \\
\hline $\mathrm{Fe}$ & $\%$ & 2.28 & 1.69 & 1.55 & 0.18 & 0.45 & 2.73 & 2.36 & 1.76 & 1.70 & 2.29 \\
\hline $\mathrm{Ga}$ & $\mu \mathrm{g} / \mathrm{g}$ & 10.7 & 7.8 & 8.3 & $<0.1$ & 1.9 & 15.4 & 13.7 & 11.4 & 11.7 & 13.7 \\
\hline $\mathrm{Gd}$ & $\mu \mathrm{g} / \mathrm{g}$ & 5.60 & 6.30 & 3.50 & 0.56 & 0.35 & 6.12 & 6.25 & 6.63 & 6.65 & 6.28 \\
\hline $\mathrm{Ge}$ & $\mu \mathrm{g} / \mathrm{g}$ & 0.12 & 0.11 & 0.09 & 0.05 & $<0.05$ & 0.12 & 0.13 & 0.11 & 0.12 & 0.10 \\
\hline Hf & $\mu \mathrm{g} / \mathrm{g}$ & 2.7 & 2.2 & 1.3 & 0.1 & 0.3 & 3.8 & 3.3 & 3.0 & 3.0 & 3.4 \\
\hline Нo & $\mu \mathrm{g} / \mathrm{g}$ & 0.97 & 1.15 & 0.52 & 0.09 & 0.06 & 1.02 & 1.05 & 1.15 & 1.22 & 1.10 \\
\hline In & $\mu \mathrm{g} / \mathrm{g}$ & 0.038 & 0.026 & 0.029 & $<0.005$ & 0.010 & 0.034 & 0.034 & 0.028 & 0.029 & 0.032 \\
\hline $\mathrm{K}$ & $\%$ & 1.53 & 0.97 & 1.22 & 0.06 & 0.28 & 1.26 & 1.19 & 1.03 & 1.01 & 1.10 \\
\hline $\mathrm{La}$ & $\mu \mathrm{g} / \mathrm{g}$ & 42.6 & 50.4 & 29.4 & 4.3 & 4.0 & 48.6 & 48.0 & 49.1 & 51.1 & 49.1 \\
\hline $\mathrm{Li}$ & $\mu \mathrm{g} / \mathrm{g}$ & 27.2 & 19.8 & 23.2 & 1.6 & 5.3 & 30.8 & 28.4 & 25.3 & 23.7 & 26.7 \\
\hline $\mathrm{Lu}$ & $\mu \mathrm{g} / \mathrm{g}$ & 0.41 & 0.48 & 0.21 & 0.03 & 0.03 & 0.37 & 0.40 & 0.46 & 0.50 & 0.46 \\
\hline $\mathrm{Mg}$ & $\%$ & 0.95 & 0.71 & 0.64 & 0.10 & 0.11 & 0.92 & 0.83 & 0.73 & 0.80 & 0.84 \\
\hline $\mathrm{Mn}$ & $\mu \mathrm{g} / \mathrm{g}$ & 2590 & 4300 & 3570 & 30 & 43 & 2840 & 3400 & 3890 & 3150 & 4400 \\
\hline Mo & $\mu \mathrm{g} / \mathrm{g}$ & 0.42 & 0.32 & 0.38 & 0.27 & 0.27 & 0.40 & 0.32 & 0.33 & 0.34 & 0.31 \\
\hline $\mathrm{Na}$ & $\%$ & 0.55 & 0.50 & 0.21 & 0.02 & 0.02 & 1.38 & 1.18 & 0.95 & 0.97 & 1.25 \\
\hline $\mathrm{Nb}$ & $\mu \mathrm{g} / \mathrm{g}$ & 8.5 & 6.3 & 5.3 & 0.3 & 1.1 & 9.1 & 8.7 & 7.6 & 7.7 & 8.5 \\
\hline $\mathrm{Nd}$ & $\mu \mathrm{g} / \mathrm{g}$ & 31.7 & 37.0 & 22.1 & 4.5 & 3.6 & 38.7 & 37.3 & 35.1 & 34.6 & 33.1 \\
\hline $\mathrm{Ni}$ & $\mu \mathrm{g} / \mathrm{g}$ & 19.5 & 11.1 & 11.4 & $<0.2$ & 2.3 & 19.5 & 19.7 & 14.4 & 14.4 & 16.8 \\
\hline $\mathrm{P}$ & $\mu \mathrm{g} / \mathrm{g}$ & 570 & 510 & 1360 & 540 & 150 & 540 & 630 & 1030 & 710 & 620 \\
\hline $\mathrm{Pb}$ & $\mu \mathrm{g} / \mathrm{g}$ & 15.5 & 11.1 & 12.9 & 1.8 & 1.9 & 14.2 & 12.9 & 11.7 & 12.0 & 12.6 \\
\hline $\operatorname{Pr}$ & $\mu \mathrm{g} / \mathrm{g}$ & 8.51 & 9.68 & 6.04 & 1.20 & 0.91 & 10.23 & 9.93 & 9.04 & 9.07 & 9.02 \\
\hline $\mathrm{Rb}$ & $\mu \mathrm{g} / \mathrm{g}$ & 75.1 & 51.9 & 65.1 & 3.7 & 19.1 & 73.8 & 68.0 & 61.7 & 67.7 & 64.4 \\
\hline $\mathrm{Re}$ & $\mu \mathrm{g} / \mathrm{g}$ & $<0.002$ & $<0.002$ & $<0.002$ & $<0.002$ & $<0.002$ & $<0.002$ & $<0.002$ & $<0.002$ & $<0.002$ & $<0.002$ \\
\hline $\mathrm{S}$ & $\%$ & 0.01 & 0.01 & 0.02 & 0.05 & 0.01 & 0.01 & 0.01 & 0.02 & 0.02 & 0.01 \\
\hline $\mathrm{Sb}$ & $\mu \mathrm{g} / \mathrm{g}$ & 0.43 & 0.30 & 0.34 & 0.17 & 0.08 & 0.35 & 0.34 & 0.26 & 0.28 & 0.31 \\
\hline $\mathrm{Sc}$ & $\mu \mathrm{g} / \mathrm{g}$ & 7.5 & 5.9 & 5.6 & 0.8 & 2.0 & 7.1 & 6.9 & 5.9 & 5.7 & 6.4 \\
\hline $\mathrm{Se}$ & $\mu \mathrm{g} / \mathrm{g}$ & $<1$ & $<1$ & $<1$ & $<1$ & $<1$ & $<1$ & $<1$ & $<1$ & $<1$ & $<1$ \\
\hline $\mathrm{Sm}$ & $\mu \mathrm{g} / \mathrm{g}$ & 5.87 & 6.65 & 4.34 & 0.87 & 0.64 & 6.77 & 6.69 & 6.79 & 6.70 & 6.49 \\
\hline $\mathrm{Sn}$ & $\mu \mathrm{g} / \mathrm{g}$ & 1.4 & 1.0 & 1.2 & $<0.2$ & 0.4 & 1.4 & 1.4 & 1.2 & 1.2 & 1.2 \\
\hline $\mathrm{Sr}$ & $\mu \mathrm{g} / \mathrm{g}$ & 255 & 166.5 & 91.8 & 878 & 630 & 231 & 214 & 226 & 222 & 236 \\
\hline $\mathrm{Ta}$ & $\mu \mathrm{g} / \mathrm{g}$ & 0.56 & 0.42 & 0.39 & $<0.05$ & 0.07 & 0.63 & 0.60 & 0.52 & 0.50 & 0.56 \\
\hline $\mathrm{Tb}$ & $\mu \mathrm{g} / \mathrm{g}$ & 0.83 & 0.95 & 0.49 & 0.08 & 0.05 & 0.89 & 0.89 & 0.95 & 0.98 & 0.90 \\
\hline
\end{tabular}


Table 2. (continued)

\begin{tabular}{|c|c|c|c|c|c|c|c|c|c|c|c|}
\hline \multicolumn{2}{|c|}{ "Sample No. } & \multirow{2}{*}{$\begin{array}{c}\text { Y016-DH1 } \\
<0.05\end{array}$} & \multirow{2}{*}{$\begin{array}{c}\text { Y039-DH1 } \\
<0.05\end{array}$} & \multirow{2}{*}{$\begin{array}{c}\text { Y049-DH1 } \\
<0.05\end{array}$} & \multirow{2}{*}{$\begin{array}{c}\text { Y103-DH3 } \\
<0.05\end{array}$} & \multirow{2}{*}{$\begin{array}{c}\text { Y103-DH4 } \\
<0.05\end{array}$} & \multirow{2}{*}{$\begin{array}{c}\text { Y002-DH1 } \\
<0.05\end{array}$} & \multirow{2}{*}{$\begin{array}{c}\text { Y002-DH2 } \\
<0.05\end{array}$} & \multirow{2}{*}{$\begin{array}{c}\text { Y002-DH3 } \\
<0.05\end{array}$} & \multirow{2}{*}{$\begin{array}{c}\text { Y002-DH4 } \\
<0.05\end{array}$} & \multirow{2}{*}{$\begin{array}{c}\text { Y002-DH5 } \\
<0.05\end{array}$} \\
\hline $\mathrm{Te}$ & $\mu \mathrm{g} / \mathrm{g}$ & & & & & & & & & & \\
\hline Th & $\mu \mathrm{g} / \mathrm{g}$ & 7.44 & 5.49 & 5.94 & 0.59 & 0.97 & 7.39 & 7.02 & 6.21 & 6.00 & 6.44 \\
\hline $\mathrm{Ti}$ & $\%$ & 0.222 & 0.171 & 0.136 & 0.009 & 0.028 & 0.280 & 0.261 & 0.215 & 0.205 & 0.246 \\
\hline $\mathrm{Tl}$ & $\mu \mathrm{g} / \mathrm{g}$ & 0.41 & 0.27 & 0.33 & 0.02 & 0.08 & 0.39 & 0.36 & 0.34 & 0.38 & 0.35 \\
\hline $\mathrm{Tm}$ & $\mu \mathrm{g} / \mathrm{g}$ & 0.40 & 0.49 & 0.21 & 0.03 & 0.03 & 0.39 & 0.40 & 0.47 & 0.50 & 0.44 \\
\hline $\mathrm{U}$ & $\mu \mathrm{g} / \mathrm{g}$ & 2.80 & 1.78 & 4.27 & 0.21 & 0.31 & 2.24 & 2.82 & 6.71 & 4.79 & 2.86 \\
\hline $\mathrm{V}$ & $\mu \mathrm{g} / \mathrm{g}$ & 58 & 44 & 43 & $<5$ & 19 & 77 & 68 & 62 & 60 & 70 \\
\hline $\mathrm{W}$ & $\mu \mathrm{g} / \mathrm{g}$ & 1.0 & 0.7 & 0.8 & 0.2 & 0.1 & 0.9 & 0.9 & 0.8 & 0.7 & 0.8 \\
\hline $\mathrm{Y}$ & $\mu \mathrm{g} / \mathrm{g}$ & 33.2 & 39.9 & 17.4 & 2.1 & 1.2 & 34.5 & 35.9 & 41.5 & 44.5 & 40.3 \\
\hline $\mathrm{Yb}$ & $\mu \mathrm{g} / \mathrm{g}$ & 2.52 & 3.04 & 1.30 & 0.19 & 0.20 & 2.40 & 2.51 & 2.87 & 3.18 & 2.81 \\
\hline $\mathrm{Zn}$ & $\mu \mathrm{g} / \mathrm{g}$ & 55 & 38 & 41 & 4 & 14 & 65 & 70 & 43 & 45 & 58 \\
\hline $\mathrm{Zr}$ & $\mu \mathrm{g} / \mathrm{g}$ & 111 & 87 & 56 & 3 & 10 & 159 & 134 & 119 & 116 & 143 \\
\hline \multicolumn{2}{|c|}{ Sample No. } & Y002-DH6 & Y002-DH7 & Y002-DH8 & Y002-DH9 & Y002-DH10 & Y002-DH11 & Y002-DH12 & Y002-DH13 & Y002-DH14 & Y002-DH15 \\
\hline $\mathrm{Ag}$ & $\mu \mathrm{g} / \mathrm{g}$ & 0.07 & 0.07 & 0.06 & 0.05 & 0.07 & 0.06 & 0.06 & 0.07 & 0.05 & 0.06 \\
\hline $\mathrm{Al}$ & $\%$ & 5.21 & 4.42 & 5.21 & 4.74 & 5.02 & 4.96 & 4.76 & 5.06 & 5.88 & 5.41 \\
\hline As & $\mu \mathrm{g} / \mathrm{g}$ & 13.1 & 6.6 & 6.5 & 6.2 & 6.8 & 6.2 & 6.3 & 6.6 & 7.8 & 6.7 \\
\hline $\mathrm{Ba}$ & $\mu \mathrm{g} / \mathrm{g}$ & 407 & 380 & 425 & 363 & 392 & 414 & 486 & 367 & 444 & 391 \\
\hline $\mathrm{Be}$ & $\mu \mathrm{g} / \mathrm{g}$ & 1.50 & 1.37 & 1.51 & 1.49 & 1.47 & 1.33 & 1.43 & 1.48 & 1.61 & 1.51 \\
\hline $\mathrm{Bi}$ & $\mu \mathrm{g} / \mathrm{g}$ & 0.13 & 0.10 & 0.11 & 0.10 & 0.12 & 0.11 & 0.12 & 0.12 & 0.14 & 0.14 \\
\hline $\mathrm{Ca}$ & $\%$ & 16.70 & 19.00 & 16.10 & 18.65 & 16.55 & 16.45 & 16.85 & 17.00 & 12.80 & 15.70 \\
\hline $\mathrm{Cd}$ & $\mu \mathrm{g} / \mathrm{g}$ & 3.14 & 2.04 & 1.76 & 1.34 & 1.70 & 2.33 & 1.55 & 1.54 & 1.67 & 3.01 \\
\hline $\mathrm{Ce}$ & $\mu \mathrm{g} / \mathrm{g}$ & 104.9 & 77.3 & 93.1 & 93.5 & 88.7 & 93.2 & 96.6 & 92.3 & 78.2 & 85.5 \\
\hline Co & $\mu \mathrm{g} / \mathrm{g}$ & 10.9 & 9.6 & 10.2 & 10.1 & 10.8 & 10.2 & 9.9 & 10.3 & 13.1 & 11.7 \\
\hline $\mathrm{Cr}$ & $\mu \mathrm{g} / \mathrm{g}$ & 64 & 36 & 48 & 43 & 49 & 56 & 50 & 49 & 40 & 37 \\
\hline Cs & $\mu \mathrm{g} / \mathrm{g}$ & 4.29 & 3.64 & 4.17 & 3.81 & 3.92 & 4.71 & 3.91 & 3.96 & 4.83 & 4.45 \\
\hline $\mathrm{Cu}$ & $\mu \mathrm{g} / \mathrm{g}$ & 15.6 & 13.8 & 15.6 & 15.3 & 16.1 & 16.7 & 14.7 & 15.7 & 15.5 & 16.4 \\
\hline Dy & $\mu \mathrm{g} / \mathrm{g}$ & 6.48 & 5.06 & 5.59 & 6.05 & 5.45 & 6.24 & 6.43 & 6.21 & 4.15 & 4.68 \\
\hline $\mathrm{Er}$ & $\mu \mathrm{g} / \mathrm{g}$ & 3.46 & 3.03 & 3.08 & 3.49 & 3.13 & 3.79 & 3.95 & 3.45 & 2.24 & 2.56 \\
\hline $\mathrm{Eu}$ & $\mu \mathrm{g} / \mathrm{g}$ & 2.00 & 1.46 & 1.59 & 1.80 & 1.63 & 1.75 & 1.94 & 1.75 & 1.28 & 1.35 \\
\hline $\mathrm{Fe}$ & $\%$ & 2.16 & 1.78 & 2.08 & 1.96 & 2.16 & 2.06 & 2.18 & 2.20 & 2.80 & 2.51 \\
\hline $\mathrm{Ga}$ & $\mu \mathrm{g} / \mathrm{g}$ & 14.1 & 11.6 & 13.5 & 12.4 & 13.4 & 13.2 & 13.1 & 12.9 & 15.5 & 14.5 \\
\hline $\mathrm{Gd}$ & $\mu \mathrm{g} / \mathrm{g}$ & 8.38 & 5.89 & 6.40 & 7.46 & 6.83 & 7.24 & 7.72 & 7.29 & 5.12 & 5.39 \\
\hline $\mathrm{Ge}$ & $\mu \mathrm{g} / \mathrm{g}$ & 0.14 & 0.10 & 0.12 & 0.14 & 0.12 & 0.14 & 0.15 & 0.13 & 0.12 & 0.12 \\
\hline Hf & $\mu \mathrm{g} / \mathrm{g}$ & 3.6 & 3.0 & 4.5 & 3.3 & 3.5 & 3.8 & 3.5 & 3.4 & 3.5 & 3.4 \\
\hline Но & $\mu \mathrm{g} / \mathrm{g}$ & 1.35 & 1.09 & 1.12 & 1.27 & 1.17 & 1.32 & 1.41 & 1.28 & 0.85 & 0.95 \\
\hline In & $\mu \mathrm{g} / \mathrm{g}$ & 0.037 & 0.029 & 0.038 & 0.033 & 0.034 & 0.037 & 0.035 & 0.038 & 0.040 & 0.039 \\
\hline $\mathrm{K}$ & $\%$ & 1.26 & 1.03 & 1.30 & 1.18 & 1.21 & 1.24 & 1.15 & 1.22 & 1.35 & 1.30 \\
\hline $\mathrm{La}$ & $\mu \mathrm{g} / \mathrm{g}$ & 68.5 & 44.9 & 56.5 & 57.8 & 54.5 & 62.2 & 60.5 & 57.0 & 41.6 & 46.4 \\
\hline $\mathrm{Li}$ & $\mu \mathrm{g} / \mathrm{g}$ & 28.6 & 23.8 & 25.6 & 26.1 & 27.0 & 27.2 & 24.3 & 26.4 & 34.6 & 30.5 \\
\hline $\mathrm{Lu}$ & $\mu \mathrm{g} / \mathrm{g}$ & 0.52 & 0.46 & 0.43 & 0.47 & 0.48 & 0.54 & 0.57 & 0.47 & 0.33 & 0.37 \\
\hline $\mathrm{Mg}$ & $\%$ & 0.87 & 0.81 & 0.81 & 0.74 & 0.82 & 0.81 & 0.82 & 0.79 & 0.96 & 0.89 \\
\hline $\mathrm{Mn}$ & $\mu \mathrm{g} / \mathrm{g}$ & 3800 & 3620 & 3330 & 3490 & 3970 & 4450 & 3980 & 4220 & 4110 & 4770 \\
\hline Mo & $\mu \mathrm{g} / \mathrm{g}$ & 0.43 & 0.33 & 0.35 & 0.29 & 0.32 & 0.23 & 0.30 & 0.29 & 0.34 & 0.32 \\
\hline $\mathrm{Na}$ & $\%$ & 0.97 & 1.02 & 1.07 & 0.95 & 1.02 & 0.93 & 0.97 & 1.02 & 1.17 & 0.99 \\
\hline $\mathrm{Nb}$ & $\mu \mathrm{g} / \mathrm{g}$ & 9.3 & 7.9 & 9.3 & 8.2 & 9.1 & 9.9 & 9.0 & 8.7 & 10.1 & 9.3 \\
\hline $\mathrm{Nd}$ & $\mu \mathrm{g} / \mathrm{g}$ & 46.5 & 32.2 & 37.2 & 40.2 & 39.6 & 40.7 & 42.3 & 39.4 & 31.4 & 32.3 \\
\hline $\mathrm{Ni}$ & $\mu \mathrm{g} / \mathrm{g}$ & 21.1 & 15.5 & 19.7 & 19.6 & 21.3 & 19.5 & 17.7 & 21.5 & 25.1 & 22.5 \\
\hline $\mathrm{P}$ & $\mu \mathrm{g} / \mathrm{g}$ & 2400 & 650 & 1020 & 900 & 840 & 710 & 690 & 940 & 890 & 910 \\
\hline $\mathrm{Pb}$ & $\mu \mathrm{g} / \mathrm{g}$ & 14.4 & 12.1 & 15.0 & 12.9 & 13.1 & 13.5 & 13.1 & 12.7 & 15.0 & 14.2 \\
\hline
\end{tabular}


Table 2. (continued)

\begin{tabular}{|c|c|c|c|c|c|c|c|c|c|c|c|}
\hline 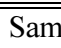 & e No. & "Y002-DH6 & "Y002-DH7 & "Y002-DH8 & "Y002-DH9 & $\overline{Y \text { Y002-DH1C }}$ & Y002-DH11 & Y002-DH12 & Y002-DH13 & $\overline{\text { Y002-DH14 }}$ & $\overline{\text { Y002-DH15 }}$ \\
\hline $\operatorname{Pr}$ & $\mu \mathrm{g} / \mathrm{g}$ & 11.98 & 8.43 & 9.68 & 10.28 & 10.55 & 10.78 & 10.98 & 10.20 & 8.57 & 9.33 \\
\hline $\mathrm{Rb}$ & $\mu \mathrm{g} / \mathrm{g}$ & 74.4 & 66.9 & 78.4 & 69.2 & 71.4 & 81.3 & 70.5 & 68.8 & 81.1 & 71.2 \\
\hline $\mathrm{Re}$ & $\mu \mathrm{g} / \mathrm{g}$ & $<0.002$ & $<0.002$ & $<0.002$ & $<0.002$ & $<0.002$ & $<0.002$ & $<0.002$ & $<0.002$ & $<0.002$ & $<0.002$ \\
\hline $\mathrm{S}$ & $\%$ & 0.01 & 0.01 & 0.01 & 0.01 & 0.01 & 0.01 & 0.01 & 0.01 & $<0.01$ & $<0.01$ \\
\hline $\mathrm{Sb}$ & $\mu \mathrm{g} / \mathrm{g}$ & 0.36 & 0.29 & 0.35 & 0.31 & 0.36 & 0.34 & 0.33 & 0.36 & 0.41 & 0.38 \\
\hline $\mathrm{Sc}$ & $\mu \mathrm{g} / \mathrm{g}$ & 7.8 & 5.8 & 7.4 & 7.1 & 7.1 & 7.1 & 6.5 & 7.3 & 8.0 & 7.7 \\
\hline $\mathrm{Se}$ & $\mu \mathrm{g} / \mathrm{g}$ & $<1$ & $<1$ & $<1$ & $<1$ & $<1$ & $<1$ & $<1$ & $<1$ & $<1$ & $<1$ \\
\hline $\mathrm{Sm}$ & $\mu \mathrm{g} / \mathrm{g}$ & 8.72 & 6.13 & 6.89 & 7.68 & 7.09 & 7.60 & 7.98 & 7.42 & 5.68 & 6.04 \\
\hline $\mathrm{Sn}$ & $\mu \mathrm{g} / \mathrm{g}$ & 1.5 & 1.3 & 1.5 & 1.3 & 1.4 & 1.5 & 1.4 & 1.4 & 1.6 & 1.5 \\
\hline $\mathrm{Sr}$ & $\mu \mathrm{g} / \mathrm{g}$ & 213 & 231 & 225 & 205 & 210 & 200 & 203 & 206 & 203 & 191.5 \\
\hline $\mathrm{Ta}$ & $\mu \mathrm{g} / \mathrm{g}$ & 0.61 & 0.53 & 0.62 & 0.56 & 0.60 & 0.65 & 0.60 & 0.59 & 0.68 & 0.64 \\
\hline $\mathrm{Tb}$ & $\mu \mathrm{g} / \mathrm{g}$ & 1.14 & 0.86 & 0.95 & 1.04 & 0.99 & 1.05 & 1.10 & 1.08 & 0.75 & 0.80 \\
\hline $\mathrm{Te}$ & $\mu \mathrm{g} / \mathrm{g}$ & $<0.05$ & $<0.05$ & $<0.05$ & $<0.05$ & $<0.05$ & $<0.05$ & $<0.05$ & $<0.05$ & $<0.05$ & $<0.05$ \\
\hline Th & $\mu \mathrm{g} / \mathrm{g}$ & 7.22 & 6.26 & 7.88 & 6.74 & 7.14 & 7.94 & 7.15 & 6.66 & 7.50 & 7.52 \\
\hline $\mathrm{Ti}$ & $\%$ & 0.263 & 0.212 & 0.264 & 0.244 & 0.256 & 0.256 & 0.245 & 0.260 & 0.301 & 0.284 \\
\hline $\mathrm{Tl}$ & $\mu \mathrm{g} / \mathrm{g}$ & 0.40 & 0.42 & 0.40 & 0.36 & 0.40 & 0.44 & 0.38 & 0.37 & 0.44 & 0.39 \\
\hline $\mathrm{Tm}$ & $\mu \mathrm{g} / \mathrm{g}$ & 0.51 & 0.44 & 0.44 & 0.48 & 0.47 & 0.54 & 0.59 & 0.49 & 0.33 & 0.38 \\
\hline $\mathrm{U}$ & $\mu \mathrm{g} / \mathrm{g}$ & 4.34 & 4.51 & 4.10 & 4.57 & 3.86 & 3.44 & 3.50 & 3.36 & 2.08 & 3.06 \\
\hline $\mathrm{V}$ & $\mu \mathrm{g} / \mathrm{g}$ & 83 & 55 & 73 & 58 & 75 & 82 & 69 & 74 & 79 & 72 \\
\hline W & $\mu \mathrm{g} / \mathrm{g}$ & 1.0 & 0.8 & 0.9 & 0.9 & 0.9 & 0.9 & 0.9 & 0.9 & 1.0 & 1.0 \\
\hline $\mathrm{Y}$ & $\mu \mathrm{g} / \mathrm{g}$ & 49.1 & 37.6 & 36.2 & 43.8 & 42.6 & 45.5 & 50.4 & 44.8 & 27.1 & 32.9 \\
\hline $\mathrm{Yb}$ & $\mu \mathrm{g} / \mathrm{g}$ & 3.16 & 2.81 & 2.82 & 2.96 & 2.97 & 3.42 & 3.64 & 3.02 & 2.12 & 2.42 \\
\hline $\mathrm{Zn}$ & $\mu \mathrm{g} / \mathrm{g}$ & 57 & 46 & 54 & 48 & 52 & 57 & 53 & 53 & 66 & 57 \\
\hline $\mathrm{Zr}$ & $\mu \mathrm{g} / \mathrm{g}$ & 143 & 120 & 178 & 132 & 139 & 149 & 139 & 132 & 136 & 136 \\
\hline
\end{tabular}

The standardized distribution pattern of rare earth elements (REEs) (Fig. 9) shows that the standardization curve normalized to chondrite composition of the samples has a "right-dipping" shape, while that normalized to Australian Post-Archean Shale (PAAS) is flat. These two pattern diagrams reflect that the sample shows no Europium anomalies (weak Europium anomalies). The three types of samples share the same rare earth distribution pattern, but the limestone from the top of the Ziliujing Formation has significantly different rare earth element abundances contrasting that of the nodular and tubular concretions, which are approximately an order of magnitude higher. At the same time, compared with the tubular concretions, the nodular concretions also had slightly lower rare earth element contents (Fig. 9 and Table 2).

\section{Carbon and Oxygen Isotopes}

With an average value of $-6.58 \%$, the $\delta^{13} \mathrm{C}_{\mathrm{V} \text {-PDB }}$ values of the tubular concretions of this study range from -8.48 to $-4.67 \%$, while the $\delta^{13} \mathrm{C}_{\mathrm{V}-\mathrm{PDB}}$ values of the nodular concretions range from -7.18 to $-4.73 \%$. Otherwise, the $\delta^{13} \mathrm{C}_{\mathrm{V}-\mathrm{PDB}}$ values of the limestone from the top of the Ziliujing Formation are much higher (ranging from 1.48 to $5.96 \%$ ) (Table 3). The ranges of isotope values for tubular and nodular concretions and limestone samples are $\delta^{18} \mathrm{O}_{\mathrm{V}-\mathrm{PDB}}=-14.16$ to $-12.46 \%$, -11.95 to $-10.64 \%$ and -12.19 to $-11.89 \%$, respectively (Fig. 10). Moreover, there is an extremely high oxygen isotope value representing the tubular concretions (Y002-10, $\delta^{18} \mathrm{O}$ reaching as high as $+4.91 \%$ ), and the value is offset from most sample data, which may be accidental, so it is not taken into account in the following discussion.

\section{Discussion}

\section{Types of Carbonate Concretions}

As mentioned above, calcareous concretions in the study area can be divided into two types. Nodular and tubular concretions have distinct characteristics. First, the nodular concretions are mainly located above the stratification plane, which is consistent with the occurrence of the strata plane, their shape is mainly nodular, approximately centimeters in size, and the interior is uniform or filled with vein-like calcite (Figs. 3e, 3f, and 4a). The other major type is mainly tubular or columnar, with a length exceeding $1 \mathrm{~m}$. It is oblique to the surrounding rock at a high angle (vertically almost). The interiors feature conduits and are always filled with sparry and micrite calcites, cyan sands, and dark muds (Figs. 4, 5, and 6). Second, in terms of their major elements, tubular and nodular concretions have obviously different oxide compositions, such as $\mathrm{SiO}_{2}, \mathrm{TiO}_{2}, \mathrm{Al}_{2} \mathrm{O}_{3}, \mathrm{Fe}_{2} \mathrm{O}_{3}{ }^{\mathrm{T}}$, and so on (Fig. 7). In addition, the $\mathrm{CaO}$ content and LOI component of the two types of concretions are highly consistent with the $\mathrm{SiO}_{2}$ content (the correlation coefficients $(\mathrm{R})$ are -0.9973 and -0.9984 , respectively), indicating that the main loss on ignition component of the samples is carbon dioxide (Fig. 8). Additionally, the large differences in $\mathrm{CaO}$ and LOI 
Table 3. Carbon and oxygen isotopes analyzing results of samples

\begin{tabular}{|c|c|c|c|c|}
\hline Laboratorial No. & Sample No. & Morphology & $\overline{\delta^{13} \mathrm{C}_{\mathrm{V}-\mathrm{PDB}} \% 0}$ & $\overline{\delta^{18} \mathrm{O}_{\text {V-PDB }} \% 0}$ \\
\hline $119-1$ & Y010 & Limestone & 1.48 & -12.19 \\
\hline $119-2$ & Y016 & Nodular concretion & -7.18 & -10.65 \\
\hline $119-3$ & Y039 & Nodular concretion & -6.99 & -11.95 \\
\hline $119-4$ & Y049 & Nodular concretion & -4.73 & -10.64 \\
\hline $119-5$ & Y103-3 & Limestone & 5.96 & -12 \\
\hline $119-6$ & Y103-4 & Limestone & 5.79 & -11.89 \\
\hline $119-7$ & Y002-1 & Tubular concretion & -6.64 & -12.53 \\
\hline $119-8$ & Y002-2 & Tubular concretion & -4.67 & -13.54 \\
\hline $119-9$ & Y002-3 & Tubular concretion & -5.62 & -12.64 \\
\hline $119-10$ & Y002-4 & Tubular concretion & -7.08 & -12.48 \\
\hline 119-10RE & Y002-4 & Tubular concretion & -6.95 & -12.46 \\
\hline $119-11$ & Y002-5 & Tubular concretion & -6.76 & -13.56 \\
\hline $119-12$ & Y002-6 & Tubular concretion & -6.79 & -13.44 \\
\hline $119-13$ & Y002-7 & Tubular concretion & -7.23 & -13.24 \\
\hline $119-14$ & Y002-8 & Tubular concretion & -6.88 & -13.54 \\
\hline $119-15$ & Y002-9 & Tubular concretion & -8.45 & -12.71 \\
\hline $119-16$ & Y002-10 & Tubular concretion & -7.1 & 4.91 \\
\hline $119-17$ & Y002-11 & Tubular concretion & -5.41 & -13.88 \\
\hline $119-18$ & Y002-12 & Tubular concretion & -4.97 & -14.16 \\
\hline $119-19$ & Y002-13 & Tubular concretion & -8.48 & -12.73 \\
\hline $119-20$ & Y002-14 & Tubular concretion & -6.1 & -13.79 \\
\hline 119-20RE & Y002-14 & Tubular concretion & -6.21 & -13.82 \\
\hline
\end{tabular}

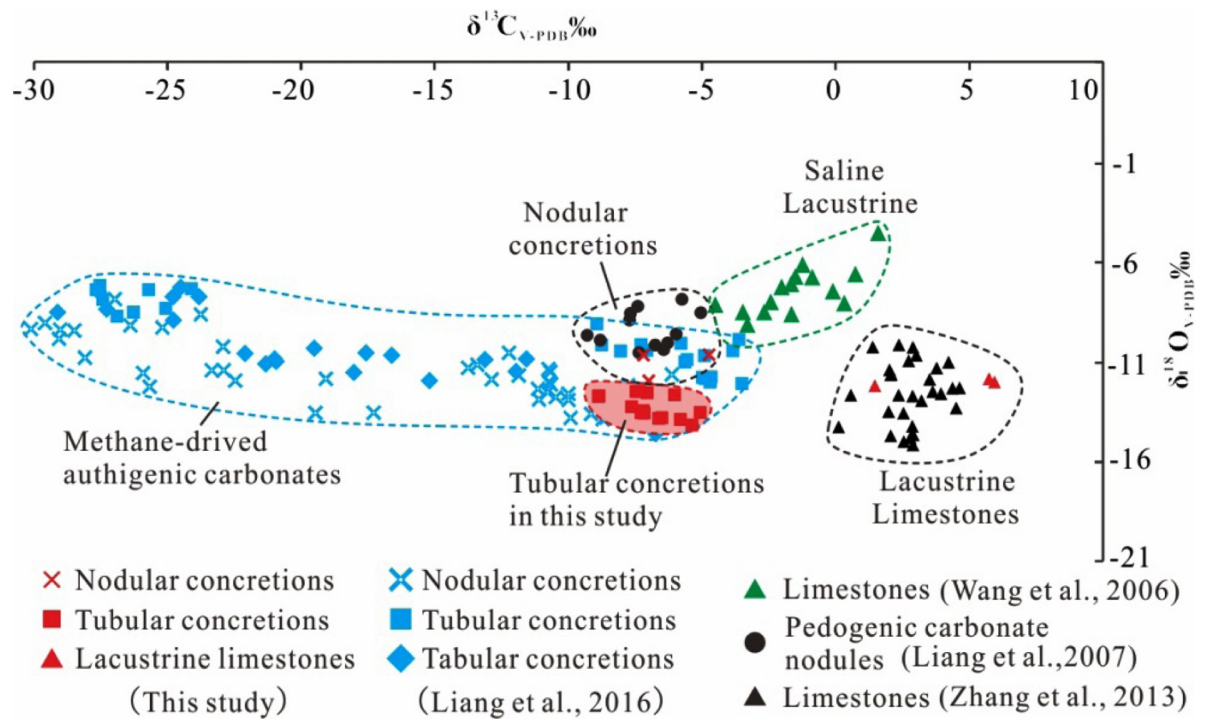

Figure 10. Cross-plot of $\delta^{13} \mathrm{C}$ and $\delta^{18} \mathrm{O}$ values.

indicate the calcium carbonate content of the two, which may imply different causes of the two types of concretions. For trace element compositions, the overall contents of the two are similar, but the nodular concretions also have a lower rare earth element content than the tubular concretions (Fig. 9). Third, compared with tubular concretions, nodular concretions have similar $\delta^{13} \mathrm{C}$ compositions, but their $\delta^{18} \mathrm{O}$ values are slightly higher. In addition, $\delta^{13} \mathrm{C}$ and $\delta^{18} \mathrm{O}$ have a weak covariation tendency (Fig. 10).
In summary, we suggest that nodular concretions and tubular concretions in the study area have different field characteristics, internal textures, and geochemical element compositions, which may be the manifestation of their different origins.

\section{Genesis of Carbonate Concretions}

A large number of studies have shown that the formation of early diagenetic calcareous concretions generally has the following four mechanisms. The first mechanism is evaporation in arid or semiarid environments (Lou and Zhao, 1993). Second, calcareous concretions are formed by the oxidation of methane when methane seeps (Aiello et al., 2001; Peckmann et al., 2001; Campbell et al., 2002; Schwartz et al., 2003; Clari et al., 2004; Tong and Chen, 2012; Ou et al., 2013; Liang et al., 2016). Third, sulfate-reducing bacteria consume sulfate to form bicarbonate in sediments rich in organic matter, ultimately resulting in calcareous tuberculosis (Gautier and Claypool, 1984; Dong et al., 2013; Pang et al., 2017). The last one, the interruption of sedimentation was promoted by underflow and ocean currents from the deep outer shelf to the oceanic basin environment, and the sediments were cemented at an early stage to form calcareous concretions (Jin, 1989).

The Sichuan Basin entered the sedimentary stage of the terrestrial 
basin in the Late Triassic, and the Jurassic-aged formation within the Sichuan Basin is famous for its "continental red beds" (Zhang et al., 2013). Therefore, considering the two types of calcareous concretions appearing in the study area, they could not be the products of undercurrents or ocean currents in a marine environment. The calcareous concretions formed by organic matter oxidized by sulfate-reducing bacteria usually develop in a strongly reducing environment and are accompanied by a large amount of framboidal pyrites (Boetius et al., 2000; Campbell et al., 2002; Jiang et al., 2006; Ou et al., 2013). In this paper, calcareous concretions mainly developed in the large-scale purple-red sandstone of the Shaximiao Formation (Fig. 3), indicating a strongly oxidizing atmosphere. This is inconsistent with the setting of a strongly reducing environment for calcareous concretions formed by the oxidation of organic matter by sulfate-reducing bacteria. In combination with the scarcity of organic matter, we could exclude the possibility of the genesis of organic matter consumed by sulfate-reducing bacteria.

Studies believe that with the progression of evaporation, ${ }^{16} \mathrm{O}$ and ${ }^{12} \mathrm{C}$ escape preferentially, resulting in a relative increase in the contents of ${ }^{18} \mathrm{O}$ and ${ }^{13} \mathrm{C}$ within the water, which leads to a simultaneous increase in the carbon and oxygen isotope values of lake water and ultimately to covariation changes in the carbon and oxygen isotopic compositions of carbonates (Liu et al., 2001). This principle can be applied in the identification of closed lake (Wang et al., 2006) and concretions originate from evaporation (Liang et al., 2007) (Fig. 10). The carbon and oxygen isotopes of nodular concretions obtained in this study show a weak simultaneous variation trend (Fig. 10). Furthermore, calcareous nodules formed by continuous intense evaporation are typically septarian concretions with inner shrinkage cracks (Li et al., 2018b), and the cracks radially narrow toward the external boundaries of the concretions (Barta, 2011). This is consistent with the characteristics of the nodular concretions in our study (Fig. 4a). Considering that nodular concretions are mainly developed in the Upper Shaximiao Formation and have a consistent relationship with stratum layers and other characteristics, we believe that the evaluated nodular concretions are caused by continuous evaporation. The formation process of the nodular concretions can be simply summarized as follows. The sedimentary strata were exposed due to drops in the surface runoff water level, and water rich in $\mathrm{CaCO}_{3}$ migrated to a relatively dry and high place from the moist area through evaporation and capillary action. During this period, the solvent water content in the infiltration solution decreased, and $\mathrm{CaCO}_{3}$ precipitated in the critical interface (Zheng et al., 2020). The incorporation of siliciclastic grains $(>20 \%)$ indicates that the growth of the nodules was proceeded by the filling of pore spaces with calcite (Zhou and Chafetz, 2009). Because of the existence of unconsolidated detrital grains, $\mathrm{CaCO}_{3}$ cemented the surrounding detrital material when precipitated. Subsequently, with the evaporation of water and the restriction of multiple factors, such as gravity and adsorption on the surface of particles (Lou and Zhao, 1993), the nodules contracted during the consolidation process and formed the nucleus of the nodular concretion. The cracks inside may be the result of seasonal wetting and drying later (Zhou and Chafetz, 2009).

Concretions formed by methane leakage usually have obvious morphological features and mostly form in the shape of mounds, nodules, tabs, chimneys, and tubes (Peckmann et al., 2001; Nyman et al., 2010; Liang et al., 2016). Chimneys and tubules are unique morphological characteristics associated with methane leakage (Pang et al., 2017). The concretions in the study area are mainly tubular and oblique to the surrounding rock at a high angle. Schwartz et al. (2003) hypothesized that methane-derived carbonates should contain a large amount of sandy and silty clastics, and the microscopic features of the tubular concretions in this study showed similar internal characteristics (Figs. 4,5 , and 6). These tubular concretions have analogous appearances and internal textures to methane-derived carbonates formed by the methane leakage process reported previously. In addition, their microscopic features are even more consistent (Campbell et al., 2002; Clari et al., 2004; Nyman et al., 2010; Liang et al., 2016). In the diagram of carbon and oxygen isotopes (Fig. 10), the isotope compositions of the samples are also consistent with methane-derived authigenic carbonates obtained by previous researchers (Liang et al., 2016). Above all, the characteristics of tubular concretions in this study may imply a methane leakage-derived genesis.

\section{Carbon Sources of Carbonate Concretions}

The oxygen isotope composition is mainly controlled by the isotopic composition of water in the sedimentary water environment and its temperature, and it is susceptible to diagenesis. The oxygen isotope values in the water of modern lakes are similar to those of groundwater at approximately $-10 \%$. It is generally believed that a $\delta^{18} \mathrm{O}$ content of carbonate minerals less than $-10 \%$ indicates that it may be affected by later diagenesis (Charreau et al., 2012). Therefore, the oxygen isotopes of the samples in this study may not represent the composition of the original deposition. Carbon isotopes, however, are less affected by diagenesis and are widely used to assess carbon sources (Liang et al., 2016). In this study, the $\delta^{13} \mathrm{C}$ contents of the nodular concretions ranged from -7.18 to $-4.73 \%$, with an average value of $-6.30 \%$. The carbon source was derived from the dissolved inorganic carbon resulting from land surface runoff according to the previous discussion, which was continuously formed by evaporation under the principle of capillary action. During this process, the carbon and oxygen isotope contents tend to increase slightly.

Studies have shown that authigenic carbonates formed during the oxidation of methane usually have negative carbon isotope compositions, generally ranging from -100 to $-30 \%$ (Whiticar et al., 1986). However, the carbon sourced from methane may be mixed with other sources, which usually induce a positive anomaly in carbon isotope analysis. For example, the dissolved inorganic carbon in seawater $\left(\delta^{13} \mathrm{C}_{\mathrm{DIC}}\right)$ may be in the range of +1.00 to $+1.30 \%$ (Irwin et al., 1977). When the two are mixed in different proportions, the carbon isotopic composition has a wide range (Liang et al., 2016). The $\delta^{13} \mathrm{C}$ values of tubular concretions in this study varied from -8.48 to $-4.67 \%$ (with an average value of $-6.58 \%$ ), indicating that the carbon source was derived from a mixture of methane and other unknown materials. The carbon source could not have been mixed with seawater because the Sichuan Basin was not a marine basin as early as the Early Jurassic period.

In the pore space within sediments where free oxygen is not available, anaerobic methane oxidation dominates $\left(\mathrm{CH}_{4}+\mathrm{SO}_{4}^{2-} \rightarrow \mathrm{HCO}-\right.$ $3+\mathrm{HS}^{-}+\mathrm{H}_{2} \mathrm{O}$; Fig. 11) (Boetius et al., 2000; Jiang et al., 2006), and the presence of bicarbonates $\left(\mathrm{HCO}_{3}^{-}\right)$leads to local supersaturation in the form of concretions. Of course, there may be a small amount of aerobic 


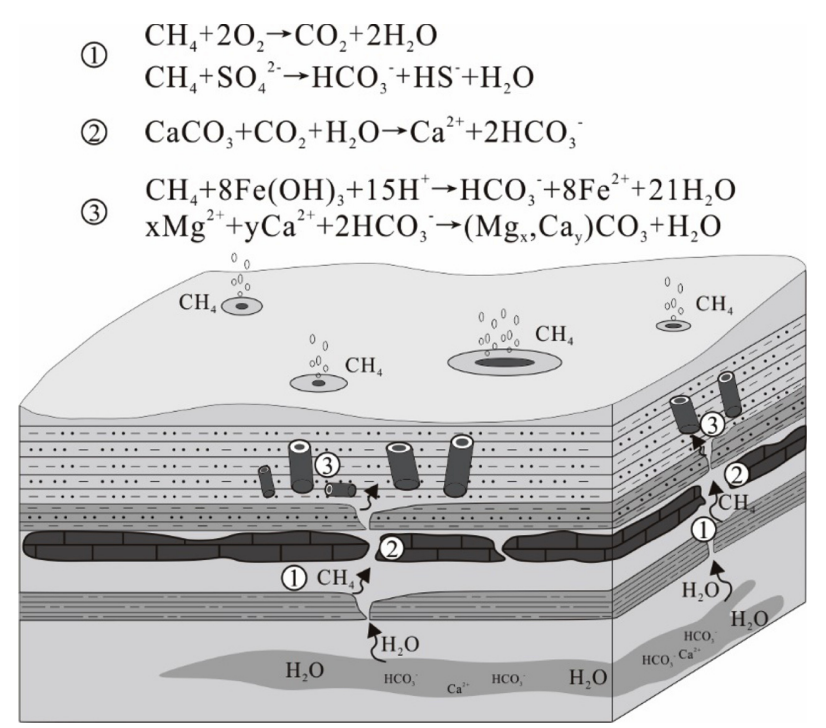

Figure 11. Formation process of the tubular concretions in the Shaximiao Formation, Yunyang (modified from Pierre et al., 2010).

oxidation reaction during methane leakage $\left(\mathrm{CH}_{4}+2 \mathrm{O}_{2} \rightarrow \mathrm{CO}_{2}+2 \mathrm{H}_{2} \mathrm{O}\right.$; Fig. 11). Carbon dioxide $\left(\mathrm{CO}_{2}\right)$, as the oxidation product of methane under aerobic conditions, dissolves in water and forms carbonic acid. As a water-soluble carbonate rock, limestone dissolves under acidic conditions and introduces carbon sources with different $\delta^{13} \mathrm{C}$ compositions (Fig. 11). The $\delta^{13} \mathrm{C}$ content of several lacustrine limestone samples collected from the Ziliujing Formation ranges from +1.48 to $+5.96 \%$, and the addition of water with bicarbonates featuring a positive carbon isotope composition may cause a positive shift in the $\delta^{13} \mathrm{C}$ composition. In combination with the geological background of the study area, we noticed the existence of gray-black shale with conchostracans on the top of the Lower Shaximiao Formation $\left(\mathrm{J}_{2} s^{1}\right)$ (Xiao et al., 2019), which indicates the presence of an intermittent lake during that period (Wang et al., 2010). Therefore, lake water with a positive $\delta^{13} \mathrm{C}$ value might be an additional source.

A large amount of liquefied muddy sediment inside the tubular concretions in the study area illustrate the addition of water and the liquefaction of the sediments. The traction deformation structures formed inside the concretions indicate that the liquefied sediments and water originate from deep strata. Marine limestone deposits with great thicknesses filled the Sichuan Basin during the Triassic, and the Early Jurassic Ziliujing Formation also contained a large number of limestone layers with positive $\delta^{13} \mathrm{C}$ compositions. Therefore, this study believes that the carbonates dissolved in the upwelling liquefied sediments and water may be an important factor driving the higher $\delta^{13} \mathrm{C}$ composition of tubular concretions. When methane enters the large-scale terrestrial red beds of the Middle Jurassic Shaximiao Formation, it is oxidized by trivalent iron oxides, which promote the formation of bicarbonates and accelerate the precipitation of calcium concretions (Fig. 11) (Smrzka et al., 2020). In summary, the higher $\delta^{13} \mathrm{C}$ composition of the tubular concretions in this study may be the result of the mixing of a methane oxidation carbon source and dissolved inorganic carbon in upwelling water. In addition, lake water with positive $\delta^{13} \mathrm{C}$ values might be an additional source.

\section{Geological Significance}

Studies have shown that methane leakage is one of the main causes of calcareous concretions, which has attracted a large amount of attention due to its close connection with gas hydrates (Clari et al., 2004; Tong and Chen, 2012; Ou et al., 2013; Liang et al., 2016). However, most of the cold-seep carbonates reported so far are located in continental margins and mid-ocean ridge spreading centers (Fig. 1a) (Campbell, 2006), and most of the cold-seep carbonate rocks found within the continent are also remnants formed in paleo-ocean environments (Clari et al., 2004; Liang et al., 2016). Although methane seeps are reported much less often in terrestrial environments than in oceanic environments, there are still some studies on sublacustrine or delta hydrothermal seeps and mud volcanism in some regions. For example, mud volcanism in the North Tanganyika Trough (Tiercelin et al., 1993), Lake Baikal (Crane et al., 1991; Golmshtok et al., 2000; Rensbergen et al., 2002), Baram Delta (Rensbergen et al., 1999) and many other places has been reported successively. Those studies, however, mainly discussed the phenomenon of ongoing methane seeps and mud volcanism based on modern geophysical methods. There are few reports focused on cold-seep carbonate rocks produced throughout geological history in a terrestrial basin. The tubular concretions generated by methane leakage reported in this paper were found in the Sichuan Basin, which was a typical terrestrial basin in the Jurassic (Li et al., 2013). Therefore, this article provides an example of the study of methane leakage-derived carbonate rocks in a typical terrestrial basin, enriching the data in the study of methane leakage in terrestrial environments.

Cold seeps are discharge sites of methane-enriched fluids and other reducing compounds (Schwartz et al., 2003). The macrogeological features of cold seeps can be expressed as methane-derived carbonate deposits (Greinert et al., 2001; Liang et al., 2016), seafloor pockmarks (Hovland and Judd, 1988) and mud volcanoes (Roberts and Carney, 1997; Clari et al., 2004). Mud volcanoes are widely distributed around the world and are cone-shaped sediment deposits formed by deep water-bearing muddy sediments ejected from the surface under high pressures within the basin (Huang et al., 2011). The formation of mud volcanoes usually requires abundant liquefied muddy sediments and a high local pressure caused by the gathering of water and gas. Finally, together with water and gas, the liquefied mud erupts and overflows triggered by some mechanism (Gao et al., 2010; He et al., 2016). Earlier studies have shown that there are large-scale liquefied sediments and muddy conglomerates in the study area (Zhong et al., 2021). The tubular concretions reported in this article indicate the emission process of methane and the fillings within the conduits illustrate that the concretions acted as transport channels for liquefied mud. Therefore, we believe that the tubular concretions in this study may be pipes formed during the discharge of $\mathrm{CH}_{4}$-rich fluids and liquefied muds and represent direct geological records of the mud volcanic plumbing system.

\section{Conclusions}

(1) There are two types of concretions developed in the Middle Jurassic Shaximiao Formation in Yunyang, Chongqing: nodular and tubular concretions. The two have notable differences in terms of their macroscopic, microscopic, and geochemical characteristics, indicat- 
ing different geneses.

(2) Nodular concretions are mainly developed in the Upper Shaximiao Formation, the interior is mostly uniform, and calcite veins are developed within some samples. These nodular concretions are formed by continuous evaporation under arid conditions.

(3) The tubular concretions developed in the Lower Shaximiao Formation are tubular and columnar in shape and obliquely cross the surrounding rock at a high angle. The conduits are generally developed inside the concretions and almost filled with calcites and argillaceous sediments. Their macroscopic, microscopic, and geochemical characteristics indicate that tubular concretions were formed by methane leakage.

(4) The tubular concretions in the study area are pipes formed during the discharge of $\mathrm{CH}_{4}$-rich fluids and liquefied sediments. They are an important part of the mud volcanic plumbing system and the most direct geological records of mud volcanic activity in the study area.

\section{Acknowledgements}

We thank the editor and reviewer for their helpful comments which improved the manuscript substantially. Also, we are grateful to Junge Qin and Yuanyin Jia for their assistance in the field and Long Xie, Nan Li, Xia Tong for their help indoor. We also thank Hui Dai, Haidong Yu, Yuxuan Zhou, Haiqian Hu, Zhenlong Zhang, Ming Xiao and other staff of the No. 208 Hydrogeological Engineering Geological Team for their help. This study was supported financially by the National Natural Science Foundation of China (41972118) and the Sixth Funding Program for the Development of Young and Middle-aged Teachers of Chengdu University of Technology (JXGG201719).

\section{References}

Aiello, I.W., Garrison, R.E., Moore, J.C., Kastner, M., and Stakes, D.S., 2001, Anatomy and origin of carbonate structures in a Miocene coldseep field. Geology, v. 29, pp. 360-364.

Barta, G., 2011. Secondary carbonates in loess-paleosoil sequences: A general review. Open Geosciences, v. 3, pp. 129-146.

Boetius, A., Ravenschlag, K., Schubert, C.J., Rickert, D., and Pfannkuche, O., 2000, A marine microbial consortium apparently mediating anaerobic oxidation of methane. Nature, v. 407, pp. 623-626.

Campbell, K.A., 2006, Hydrocarbon seep and hydrothermal vent paleoenvironments and paleontology: Past developments and future research directions. Palaeogeography, Palaeoclimatology, Palaeoecology, v. 232, pp. 362-407.

Campbell, K.A., Farmer, J.D., and Marais, D.D., 2002, Ancient hydrocarbon seeps from the Mesozoic convergent margin of California: carbonate geochemistry, fluids and palaeo-environments. Geofluids, v. 2, pp. 63-94.

Charreau, J., Kent-Corson, M.L., Barrier, L., Augier, R., Ritts, B.D., Chen, Y., France-Lannord, C., and Guilmette, C., 2012, A high-resolution stable isotopic record from the Junggar Basin (NW China): Implications for the paleotopographic evolution of the Tianshan Mountains. Earth and Planetary Science Letters, v. 341-344, pp. 158-169.

Chen, C., Yang, X.F., Wang, X.Z., Li, B., Huang, Z.S., Tang, R.F., and Du, Y., 2020, Sedimentary facies analysis of lacustrine carbonate in the Da' anzhai Member, Ziliujing Formation, Lower Jurassic, in northeastern Sichuan Basin. Geological Review, v. 66, pp. 836-852.

Chen, X.H., Zeng, J.L., Zhang, T.S., Zhu, H.H., Zhang, J.X., Li, S.X., and
LI, Y.Y., 2019, Geochemical response to paleo-climate fluctuation during Xintiangou Period (Middle Jurassic), Northeastern Sichuan. Acta Petrologica Sinica, v. 93, pp. 3223-3238.

Clari, P., Cavagna, S., Martire, L., and Hunziker, J., 2004, A Miocene mud volcano and its plumbing system: a chaotic complex revisited (Monferrato, NW Italy). Journal of Sedimentary Research, v. 74, pp. 662-676.

Conti, S., and Fontana, D., 2005, Anatomy of seep-carbonates: ancient examples from the Miocene of the northern Apennines (Italy). Palaeogeography, Palaeoclimatology, Palaeoecology, v. 227, pp. 156-175.

Crane, K., Hecker, B., and Golubev, V., 1991, Hydrothermal vents in Lake Baikal. Nature, v. 350, pp. 281-281.

Dong, J., Zhang, S., Jiang, G., Li, H., and Gao, R., 2013, Greigite from carbonate concretions of the Ediacaran Doushantuo Formation in South China and its environmental implications. Precambrian Research, v. 225, pp. 77-85.

Gao, Y.J., Yang, L., Han, W.M., and Hu, B., 2010, Formation mechanism of mud diaper and mud volcano in Niger delta. Xinjiang Petroleum Geology, v. 31, pp. 332-334.

Gautier, D.L., and Claypool, G.E., 1984, Interpretation of methanic diagenesis in ancient sediments by analogy with processes in modern diagenetic environments, in McDonald, D.A., and Surdam, R.C., eds., Modern diagenetic environments: Tulsa, Memoir-American Association of Petroleum Geologists, v. 32, pp. 111-123.

Golmshtok, A.Y., Duchkov, A.D., Hutchinson, D.R., and Khanukaev, S.B., 2000, Heat flow and gas hydrates of the Baikal Rift Zone. International Journal of Earth Sciences, v. 89, pp. 193-211.

Greinert, J., Bohrmann, G., and Suess, E., 2001, Gas hydrate-associated carbonates and methane-venting at Hydrate Ridge: classification, distribution, and origin of authigenic lithologies. Natural gas hydrates: occurrence, distribution, and detection. Geophysical Monograph, v. 124, pp. 99-113.

Guo, Z.W., Deng, K.L., and Han, Y.H., 1996, The formation and development of Sichuan Basin. Geological Publish House, Beijing, 200 p.

He, Y., Zhu, L.D., Yang, W.G., Chen, Q., Tao, G., and Xie, L., 2016, First discovery of mud volcanoes in the East of Gaize, Tibet. Geological Science and Technology Information, v. 35, pp. 38-42.

Hovland, M., and Judd, A.G., 1988, Seabed pockmarks and seepages: impact on geology, biology and the marine environment. Graham and Trotman, London, $293 \mathrm{p}$.

Huang, H.G., Di, P.F., and Chen, D.F., 2011, Global distribution and research progress of mud volcanoes. Bulletin of Mineralogy, Petrology and Geochemistry, v. 30, pp. 189-197.

Irwin, H., Curtis, C., and Coleman, M., 1977, Isotopic evidence for source of diagenetic carbonates formed during burial of organic-rich sediments. Nature, v. 269, pp. 209-213.

Jiang, G.Q., Shi, X.Y., and Zhang, S.H., 2006, Methane seeps, methane hydrate destabilization, and the Late Neoproterozoic postglacial cap carbonates. Chinese Science Bulletin, v. 51, pp. 1152-1173.

Jin, R.G., 1989, A deep water sedimentary criteria-“Knotty Nodule"and origin. Acta Sedimentologica Sinica, v. 7, pp. 51-61.

Li, Y.Q., He, D.F., Li, D., Lu, R.Q., Fan, C., Sun, Y.P., and Huang, H.Y., 2018a, Sedimentary provenance constraints on the Jurassic to Cretaceous paleogeography of Sichuan Basin, SW China. Gondwana Research, v. 60, pp. 15-33.

Li, Y.R., Zhang, W.W., Aydin, A., and Deng, X.H., 2018b, Formation of calcareous nodules in loess-paleosol sequences: Reviews of existing models with a proposed new "per evapotranspiration model". Journal of Asian Earth Sciences, v. 154, pp. 8-16.

Liang, B., Wang, Q.W., and Kan, Z.Z., 2007, Carbon and oxygen isotopic compositions of carbonate nodule in the Shaximiao Formation of the Middle Jurassic, Sichuan Basin. Journal of Mineralogical and Petrological Sciences, v. 27, pp. 54-58.

Liang, H.M., Chen, X., Wang, C.S., Zhao, D.K., and Weissert, H., 2016, Methane-derived authigenic carbonates of Mid-Cretaceous age in Southern Tibet: Types of carbonate concretions, carbon sources, and 
formation processes. Journal of Asian Earth Science, v. 115, pp. 153-169.

Liu, C.L., Zhao, Q.H., and Wang, P.X., 2001, Correlation between carbon and oxygen isotopic ratios of lacustrine carbonates and types of oilproducing paleolakes. Geochimica, v. 30, pp. 363-367.

Lou, Z.H., and Zhao, X.F., 1993, The origin of calcareous cores in the alluvial system of the Cang-fanggou Group. Experimental Petroleum Geollogy, v. 15 , pp. $81-85$.

Meng, Q.R., Wang, E., and Hu, J.M., 2005, Mesozoic sedimentary evolution of the Northwest Sichuan Basin: Implication for continued clockwise rotation of the South China Block. Geological Society of America Bulletin, v. 117, pp. 396-410.

Milkov, A.V., 2005, Global distribution of mud volcanoes and their significance in petroleum exploration as a source of methane in the atmosphere and hydrosphere and as a geohazard. Mud Volcanoes, Geodynamics and Seismicity, v. 51, pp. 29-34.

Nyman, S.L., Nelson, C.S., and Campbell, K.A., 2010, Miocene tubular concretions in East Coast Basin, New Zealand: Analogue for the subsurface plumbing of cold seeps. Marine Geology, v. 272, pp. 319-336.

Ou, L.H., Yi, H.S., Xia, G.Q., Qian, L.J., and Zhang, C., 2013, Origin and petroleum geological significance of carbonate rock concretes in Linxi Formation, northeast of Inner Mongolia, China. Journal of Chengdu University of Technology (Science \& Technology Edition), v. 40, pp. 417-423.

Pang, Q., Li, L., Hu, G., Tan, X.C.?Ma, T., Zhao, D.F., Lu, F.F., Chen, H.Y., and Xiong, Y., 2017, Characteristics and genetic mechanism of calcareous concretions in the Early Cambrian Qiongzhusi Formation of Northern Sichuan Basin. Acta Sedimentologica Sinica, v. 35, pp. 681-690.

Pantin, H.M., 1958, Rate of formation of a diagenetic calcareous concretion. Journal of Sedimentary Research, v. 28, pp. 366-371.

Peckmann, J., Reimer, A., Luth, U., Luth, C., Hansen, B., Heinicke, C., Hoefs, J., and Reitner, J., 2001, Methane-derived carbonates and authigenic pyrite from the northwestern Black Sea. Marine Geology, v. 177, pp. $129-150$.

Peckmann, J., and Thiel, V., 2004, Carbon cycling at ancient methane-seeps. Chemical Geology, v. 205, pp. 443-467.

Pierre, F.D., Martire, L., Natalicchio, M., Clari, P., and Petrea, C., 2010, Authigenic carbonates in Upper Miocene sediments of the Tertiary Piedmont Basin (NW Italy): Vestiges of an ancient gas hydrate stability zone? Geological Society of America Bulletin, v. 122, pp. 994-1010.

Rensbergen, P.V., Morley, C.K., Ang, D.W., Hoan, T.Q., and Lam, N.T., 1999, Structural evolution of shale diapirs from reactive rise to mud volcanism: 3D seismic data from. Journal of the Geological Society, v. 156, pp. 633-650.

Rensbergen, P.V., Batist, M.D., Klerkx, J., Hus, R., and Krinitsky, P., 2002, Sublacustrine mud volcanoes and methane seeps caused by dissociation of gas hydrates in Lake Baikal. Geology, v. 30, pp. 631-634.

Roberts, H.H., and Carney, R.S., 1997, Evidence of episodic fluid, gas, and sediment venting on the northern Gulf of Mexico continental slope. Economic Geology \& the Bulletin of the Society of Economic Geologists, v. 92, pp. 863-879.

Taylor, S.R., and McLennan, S.M., 1985, The continental crust: its composition and evolution: an examination of the geochemical record preserved in sedimentary rocks. Blackwell Scientific Publish, 169-200 p.

Schwartz, H., Sample, J., Weberling, K.D., Minisini, D., and Moore, J.C.,
2003, An ancient linked fluid migration system: cold-seep deposits and sandstone intrusions in the Panoche Hills, California, USA. Geo-Marine Letters, v. 23, pp. 340-350.

Smrzka, D., Feng, D., Himmler, T., Zwicker, J., Hu, Y., Monien, P., Tribovillard, N., Chen, D., and Peckmann, J., 2020, Trace elements in methane-seep carbonates: Potentials, limitations, and perspectives. Earth Science Reviews, v. 208, pp. 103263.

Sun, S.S., and McDonough, W.F., 1989, Chemical and isotopic systematics of oceanic basalts: implications for mantle composition and processes. Geological Society, London, Special Publications, v. 42, pp. 313-345.

Tiercelin, J.J., Boulègue, J., and Simoneit, B., 1993, Hydrocarbons, sulphides, and carbonate deposits related to sublacustrine hydrothermal seeps in the North Tanganyika Trough, East African Rift. Springer Berlin Heidelberg, 96-113 p.

Tong, H., and Chen, D., 2012, First discovery and characterizations of Late Cretaceous seep carbonates from Xigaze in Tibet, China. Chinese Science Bulletin, v. 57, pp. 4363-4372.

Wang, Q.W., Liang, B., and Kan, Z.Z., 2006, Carbon and oxygen isotopic compositions of lacustrine carbonates of the Early Jurassic Ziliujing Formation in the Sichuan Basin and their paleo limnological significance. Journal of Mineralogical and Petrological Sciences, v. 26, pp. 87-91.

Wang, Y.B., and Xu, H.J., 2001, Relations between evolution of sedimentary cycles and tectonic uplift around Sichuan Basin from Jurassic to early Cretaceous. Earth Science-Journal of China University of Geosciences, v. 26, pp. 241-246.

Wang, Y.D., Fu, B.H., Xie, X.P., Huang, Q.S., Li, K., Li, G., Liu, Z.S., Yu, J.X., Pan, Y.H., Tian, N., Jiang, Z.K., 2010, The terrestrial Triassic and Jurassic systems in the Sichuan Basin, China. University of Science \& Technology of China Press, $145-155$ p.

Whiticar, M.J., Faber, E., and Schoell, M., 1986, Biogenic methane formation in marine and freshwater environments: $\mathrm{CO}_{2}$ reduction vs. acetate fermentation-isotope evidence. Geochimica Et Cosmochimica Acta, v. 50, pp. 693-709.

Xiao, F.S., Huang, D., Zhang, B.J., Tang, D.H., Ran, Q., Tang, Q.S., Yin, H., 2019, Geochemical characteristics and geological significance of natural gas in Jurassic Shaximiao Formation, Sichuan Basin. Acta Petrolei Sinica, v. 40, pp. 568-576.

Zhang, W., Li, Z.W., Feng, F., Zhai, Z.H., Sun, W., Tang, C., Ding, Y., and Liu, S.G., 2013, Carbon and oxygen isotopic composition of lacustrine carbonate rocks of the Lower-Middle Jurassic in NE part of central Sichuan Province and their palaeo environmental significance. Journal of Palaeogeography, v. 15, pp. 247-259.

Zheng, D.S., Qi, S.S., Yang, W.T., Wang, Y.P., and Li, Y., 2020, Origin and paleoclimate significance of calcareous concretions in the upper member of the Sunjiagou Formation of Upper Permian in Yiyang area, western Henan province. Journal of Henan Polytechnic University (Natural Science), v. 39, pp. 22-31.

Zhong, Y., Zhu, L.D., Yang, W.G., Zhou, Y.X., Xiong, C., Xiao, M., Li, D.L, and Zhang, H.L., 2021, Soft-sediment deformation structures and its geological significance of the Shaximiao Formation in Yunyang, Chongqing, China. Journal of Chengdu University of Technology (Science \& Technology Edition), v. 48, pp. 165-177. 


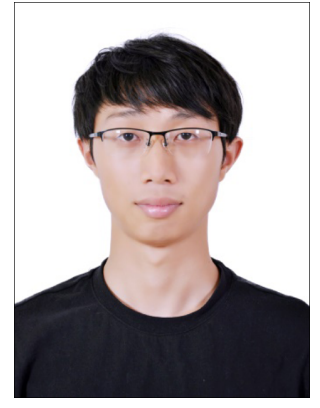

Yao Zhong is a ph.D Candidate at the Institute of Sedimentary Geology, Chengdu University of Technology. His research has been concerned with sedimentary geology and geochemistry. The research focuses on the Qinghai-Tibet Plateau and Sichuan Basin.

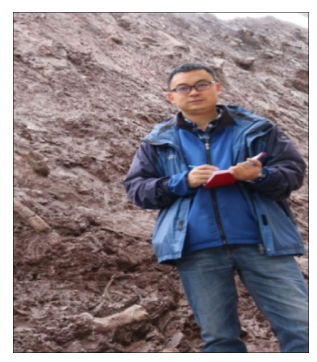

Wenguang Yang is a lecturer at Chengdu University of Technology. His research interests involve paleoclimate and palaeobios. The research focuses on the Qinghai-Tibet Plateau and Sichuan Basin.

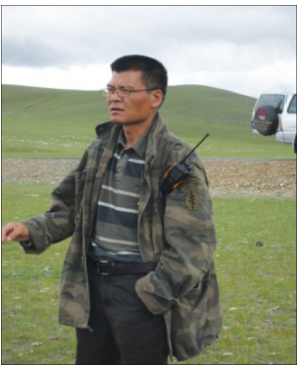

Lidong Zhu, Doctor of paleontology and stratigraphy. He is a professor of the Institute of Sedimentary Geology, Chengdu University of Technology. He is working on the regional geology of the Sichuan Basin and related teaching work.

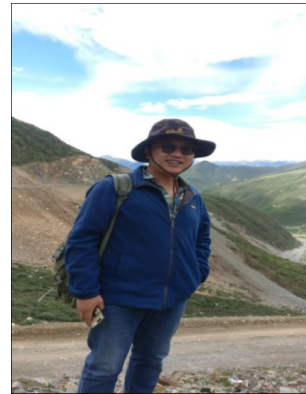

Hongliang Zhang is a ph.D Candidate of College of Earth Science at Chengdu University of Technology. His research has been concerned with sedimentary geology, geo-heritage, and geochemistry. The research focuses on the Qinghai-Tibet Plateau and Sichuan Basin.

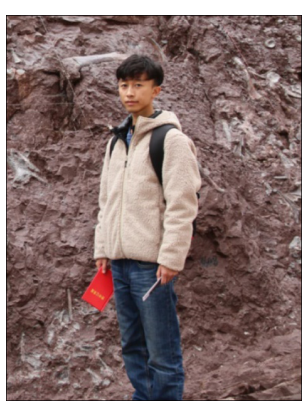

Yuanjun Mai is a ph.D Candidate at Institute of Sedimentary Geology at Chengdu University of Technology. His research has been concerned with geochronology and geochemistry. The research focuses on the Qinghai-Tibet Plateau.

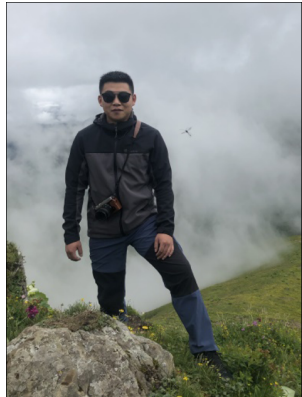

Yu Zhou, a postgraduate student of Institute of Sedimentary Geology at Chengdu University of Technology. He is majors in paleontology and stratigraphy. 